\title{
Menus for Feeding Black Holes
}

\author{
Bence Kocsis • Abraham Loeb
}

Received: 2 April 2013 / Accepted: 12 August 2013

\begin{abstract}
Black holes are the ultimate prisons of the Universe, regions of spacetime where the enormous gravity prohibits matter or even light to escape to infinity. Yet, matter falling toward the black holes may shine spectacularly, generating the strongest source of radiation. These sources provide us with astrophysical laboratories of extreme physical conditions that cannot be realized on Earth. This chapter offers a review of the basic menus for feeding matter onto black holes and discusses their observational implications.
\end{abstract}

Keywords Black holes · black hole binaries · Accretion and accretion disks · Plasmas - astrophysical

PACS 04.70.Bw $\cdot$ 04.25.dg $\cdot$ 97.60.Lf $\cdot 98.62 . \mathrm{Mw} \cdot 95.30 . \mathrm{Qd}$

\section{Introduction}

There are several avenues for feeding matter onto black holes. Black holes can accrete ambient gas from the interstellar medium or from an attached gaseous disk, they may be fed by winds of a binary companion star or directly accrete from a binary companion through Roche lobe overflow, they can directly swallow or tidally disrupt stellar objects that approach them, or they may grow by merging with other black holes. Here we review these possibilities in detail and describe the corresponding astrophysical models of accretion.

We start with an overview of the standard formation and evolutionary scenarios of the three main classes of black holes: stellar mass, intermediate mass, and supermassive black holes. In subsequent sections we discuss the main classes of accretion including spherical accretion, disk accretion, and accretion due to tidal disruptions of stars.

Bence Kocsis

Institute for Advanced Study, Princeton, NJ, USA

E-mail: bkocsis@ias.edu

Abraham Loeb

Harvard-Smithsonian Center for Astrophysics, Cambridge, MA, USA

E-mail: aloeb@cfa.harvard.edu 


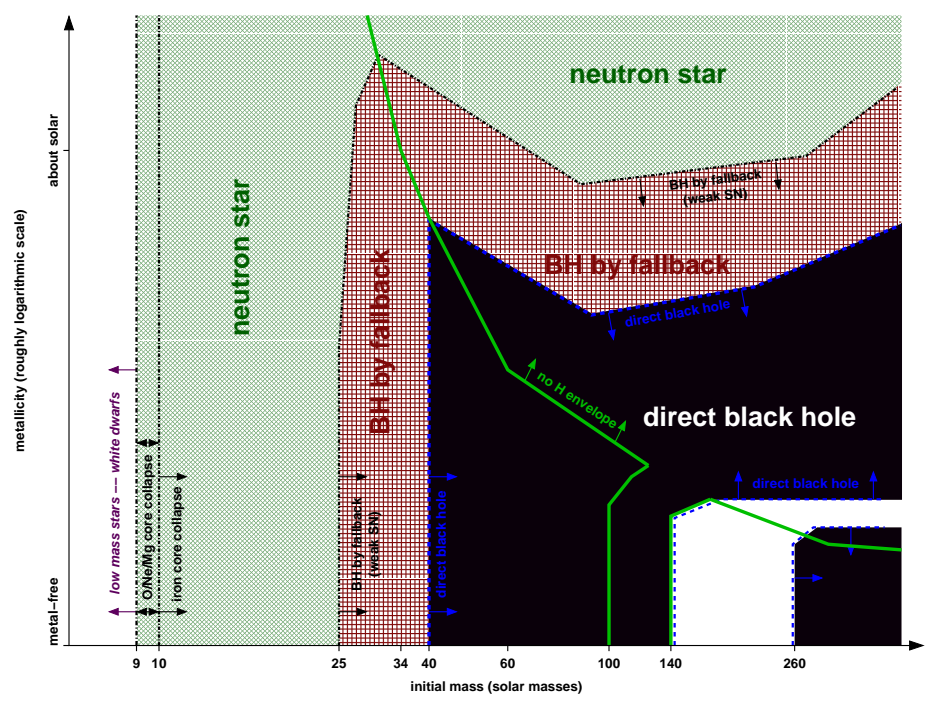

Fig. 1 Types of stellar remnants of massive stars for different initial stellar masses and metallicity. Figure credit: Heger et al. (2003).

\section{Black Hole Formation and Evolution}

\subsection{Collapse of Massive stars}

Compact objects may form when the core of a massive star consumes its nuclear fuel and loses pressure support, resulting in a catastrophic collapse. Whether the final state is a neutron star or a black hole depends on the mass of the star, its composition (i.e. metallicity) as depicted in Figure 1 and rotation speed. Stars with masses between $\sim 8-25$ often explode as a Type II supernova and leave a neutron star/pulsar remnant. For more massive stars, in the range $25-35 \mathrm{M}_{\odot}$, the fall back of the carbon-oxygen core may be significant to crush the remnant into a black hole (Fryer 1999). For even more massive stars, the complete hydrogen envelope is lost to strong winds, leaving an isolated Wolf-Rayet star. This star then explodes as a Type-Ib/c supernova. Depending on the less-understood Wolf-Rayet mass loss rates, the final remnant is either a neutron star or a black hole. Recent work (e.g., Ugliano et al. 2012) shows that black holes can form across a somewhat wider range of initial masses $\left(M \gtrsim 15 \mathrm{M}_{\odot}\right)$, and neutron stars can form from much higher mass progenitors $\left(M \gg 25 \mathrm{M}_{\odot}\right)$. This is also hinted by the young progenitors inferred for observed magnetars $\left(M>40 \mathrm{M}_{\odot}\right.$; Muno et al. 2006).

The mass of the remnant black hole depends on the amount of wind loss, fall back, and metallicity. It is typically estimated to be between $5-15 \mathrm{M}_{\odot}$ for solar metallicities and between $5-80 \mathrm{M}_{\odot}$ in globular clusters or metal-poor galaxies with $0.01 Z_{\odot}$ (Belczynski et al. 2010). Higher mass black holes may form during the collapse of the first stars, the so-called Population III stars, discussed in $\S 2.4$. 
2.2 Black Holes in Stellar Binaries

Many stellar-mass black holes and neutron stars are found in close binary systems. In the local Universe, black-hole X-ray binaries come in two flavors, depending on the mass of the companion star: low-mass $X$-ray binaries where a low-mass companion transfers mass owing to the tidal force exerted by the black hole, and high-mass $X$-ray binaries (BH-HMXB) where the companion is a massive star which could also transfer mass to the black hole through a wind. At redshifts $z \gtrsim 6$ when the age of the Universe was short, BH-HMXB were probably most important since they are known to produce their X-rays over a short lifetime $\left(\sim 10^{8} \mathrm{yr}\right)$. The cumulative X-ray emission from BH-HMXBs is expected to be proportional to the star formation rate Grimm et al. 2003; Ranalli et al. 2003; Mineo et al. 2012). If indeed the early population of stars was tilted towards high masses and binaries were common, BH-HMXB may have been more abundant per star formation rate in high redshift galaxies. The X-rays produced by $\mathrm{BH}-$ HMXBs may have had important observable effects as they catalyzed $\mathrm{H}_{2}$ formation, heated the intergalactic medium, and modified the 21-cm signal from neutral hydrogen. Their overall influence was, however, limited: hydrogen could not have been reionized by X-ray sources based on current limits on the unresolved component of the X-ray background.

The formation of $\mathrm{BH}$ binaries may be linked to the evolution of binary stars, transferring mass during their lives. X-ray binaries in particular, are believed to be affected by mass transfer in the giant phase. The standard evolutionary scenario leading to a HMXB is depicted in Fig. 2. Here at least one of the stars in the binary must be a massive star. After a few million years, it exhausts its hydrogen, expands into a red giant, and transfers its entire envelope to the secondary. At this stage, the primary and secondary become a Wolf-Rayet star and a massive O/B star. The primary then explodes as a type Ib supernova, and becomes a neutron star or a black hole depending on its initial mass (see Fig. 1). The powerful winds of the massive secondary star sources accretion for the companion compact object. Later, as the secondary runs out of hydrogen and expands to within the Roche lobe of the compact object where the gravity of the compact object dominates,

$$
r_{R}=a(1-e)\left(\frac{m}{2 M}\right)^{1 / 3}
$$

the compact object will accrete continuously from the secondary. Here $M, m, a, e$ are the primary and secondary masses, semimajor axis, and eccentricity. As the secondary becomes a red giant, it may completely engulf the orbit. In this common envelope stage, the angular momentum of the binary can quickly decrease as the envelope is heated and ejected. If the core of the giant merges with the neutron star, it may form a Thorne-Zytkow object (Thorne and Zytkow 1977), or if it is ejected completely, it leaves behind a black hole or neutron star primary orbiting closely around a WolfRayet star secondary. Next, the secondary explodes as a Type-Ib supernova. If the kick from the supernova explosion is larger than the gravitational binding energy, the two compact objects may fly apart, as single black holes or neutron stars. Alternatively, if the kick is weaker, it leaves behind a relativistic eccentric double black hole, double neutron star, or black hole-neutron star binary.

These double compact object binaries then gradually loose energy and angular momentum due to gravitational wave emission, which leads to the shrinking of the orbital separation and eccentricity. The existence of gravitational waves is a generic prediction 
T 3 Myr, N 1 $10^{4}$<smiles>C1=CC=CC2(C=C1)C=CC=CC=C2</smiles>

$\mathrm{T} \sim 10^{4} \mathrm{yr}, \mathrm{N} \sim 30$

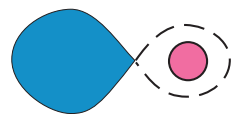

$\mathrm{T} \sim 2 \cdot 10^{5} \mathrm{yr}, \mathrm{N} \sim 500$

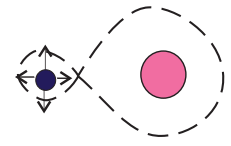

$v \sim 10^{-2} \mathrm{yr}^{-1}$

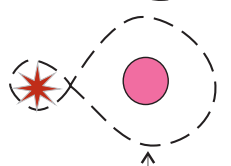

$\mathrm{T} \sim 10^{4} \mathrm{yr}, \mathrm{N} \sim 100$

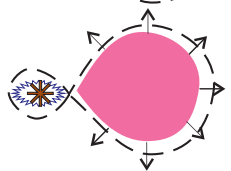

type Ib Supernova and

becomes a neutron star

or black hole

Secondary is close to Roche lobe.

Accretion of stellar wind results in

powerful X-ray emission

Helium core of the secondary

with compact companion inside

mass-losing common envelope

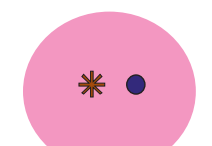

More massive star (primary)

Helium-rich WR star

with OB-companion
$\mathrm{T} \sim 2 \cdot 10^{4} \mathrm{yr}, \mathrm{N} \sim 50$

Wolf-Rayet star with compact companion surrounded by expanding envelope $10^{4} \mathrm{yr}, \mathrm{N} \sim 30$

Secondary explodes as type

Ib Supernova, $v \sim 10^{-4} \mathrm{yr}^{-1}$

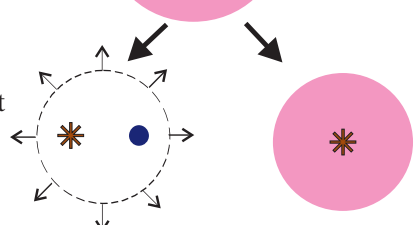

Components merge

Red (super)giant with neutron

star or black hole core

(Thorne-Zytkow object)

T 10 Gyr, N 10

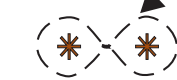

米等

$\mathrm{T} \sim 10$ Gyr, N 10

* Single neutron star

or black hole

Binary relativistic

star

Merger of components

with a burst of emission

of gravitational waves,

53

erg, $v \sim 10^{-5} \mathrm{yr}^{-1}$

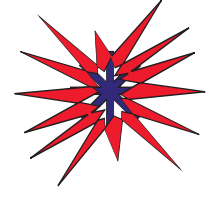

Supernova explosion

disrupts the system.

Two single neutron

stars or black holes

Fig. 2 Evolutionary stages of high mass binary stars from top to bottom. The initially more massive star (primary) is shown on the left. A black hole or neutron star forms in the $4^{\text {th }}$ stage during a type Ib supernova possibly accompanied by a long GRB. The $5^{\text {th }}$ stage represents a typical HMXB. Once the secondary fills its Roche lobe, it may supply super-Eddington accretion and lead to a super-soft X-ray source. Later the binary inspirals in a common envelope, ejecting the envelope. This may lead to a second supernova, leaving a relativistic double $\mathrm{BH}$, NS, or BH/NS binary. These objects merge after gravitational wave emission, possibly generating a short GRB. The approximate timescale and number of such binaries in the Galaxy are labeled in each evolutionary stage. Figure credit: Postnov and Yungelson (2006). 
of Einstein's theory of gravity. They represent ripples in space-time generated by the motion of the two black holes as they move around their common center of mass in a tight binary. The energy carried by the waves is taken away from the kinetic energy of the binary, which therefore tightens with time. Ultimately, the gravitational waves emitted close to merger will be detectable by Advanced LIGQ1 1 and VIRGd2. Additionally, if at least one of the components is a neutron star, the merger may produce a bright gamma ray burst (GRB).

The above scenario involves massive stars. The analogous evolutionary scenario for less massive binary stars leads to a white dwarf, neutron star, or black hole with a moderate to low-mass main sequence star, and ultimately a double compact object binary (e.g. double degenerate white dwarf binary). In this case, the separation is much larger larger than commonly observed in low mass X-ray binaries (LMXBs). The formation of LMXBs is less understood. Many-body interactions, especially in globular clusters may lead to LMXBs. Alternatively, a massive binary system may form LMXBs if there is a rapid super-Eddington wind phase and angular momentum evolution in between the $5^{\text {th }}$ and $6^{\text {th }}$ stage in Figure 2 Black holes may form in low mass binaries during an accretion-induced collapse if a neutron star is feeding through Roche lobe overflow from a companion (Dermer and Atovan 2006). Another possibility is the merger of a WD with a NS (Paschalidis et al. 2009), although in this case it is unclear whether sufficient mass will accrete onto the NS if the WD material undergoes explosive nuclear burning (Fernández and Metzger 2013).

Neutron star or black hole binaries that accrete from a strong wind of the companion star, may be modeled using Bondi-Hoyle-Lyttleton accretion. Compact objects that accrete from the companion star through a Roche-lobe overflow, provide examples of disk accretion. We discuss Bondi and disk accretion in $\S 3$ and $\S$ 田 respectively.

A neutron star in a LMXB that undergoes mass transfer from a secondary star is expected to be spun up during the accretion to millisecond periods, leaving a millisecond pulsar. Since black holes are more massive with a larger angular momentum, they cannot be spun up significantly by a lower mass companion.

\subsection{Supermassive black holes}

Supermassive black holes of masses between $10^{6}-10^{10} \mathrm{M}_{\odot}$ are observed in the centers of most if not all nearby galaxies. As they accrete gas, the gas shines as a bright pointlike quasi-stellar object, called a quasar. These objects are observed from cosmological distances up to high redshifts, where the Universe was less than a billion years old. How could such massive black holes form in such a short amount of time?

Growing a supermassive black hole very quickly is difficult. Accretion of collisionless dark matter particles is negligible and can be ignored (Shapiro and Teukolsky 1983a). For gas accretion - there is a maximum luminosity at which the environment of a black hole of mass $M_{\mathrm{BH}}$ may shine and still accrete gas, called the Eddington limit, $L_{\mathrm{Edd}}$. This limit is obtained by setting the outward continuum radiation pressure equal to the inward gravitational force. Denoting the gravitational potential with $\Phi$, pressure with $p$, density with $\rho$,

$$
\nabla \Phi=-\frac{\nabla p}{\rho}=\frac{\kappa}{c} \boldsymbol{F}_{\mathrm{rad}},
$$

\footnotetext{
1 http://www.ligo.caltech.edu/

2 http://www.ego-gw.it/
} 
where in the last equality we assumed that the pressure is dominated by radiation pressure which is associated to a radiation flux $\boldsymbol{F}_{\text {rad }}$. Here $\kappa$, is the opacity. There are two primary sources of opacity for the typical densities and temperatures here: Thomson electron scattering and bremsstrahlung (i.e labeled free-free absorption) with

$$
\kappa_{\mathrm{es}}=\frac{\sigma_{\mathrm{T}}}{\mathrm{m}_{\mathrm{p}}}=0.4 \mathrm{~cm}^{2} \mathrm{~g}^{-1} \quad \text { and } \kappa_{\mathrm{ff}} \approx 8 \times 10^{22} \mathrm{~cm}^{2} \mathrm{~g}^{-1}\left(\frac{\rho}{\mathrm{gcm}^{-3}}\right)\left(\frac{T}{\mathrm{~K}}\right)^{-7 / 2}
$$

where we assumed a pure hydrogen plasma for simplicity, where $\mathrm{m}_{\mathrm{p}}$ denotes proton mass and $\sigma_{\mathrm{T}}$ denotes the Thomson cross section. Substituting equation (2) into the definition of the luminosity of the source bounded by a surface $S$, we get

$$
L=\int_{S} \boldsymbol{F}_{\mathrm{rad}} \cdot \mathbf{d} \boldsymbol{S}=\frac{c}{\kappa} \int_{S} \nabla \Phi \cdot \mathbf{d} \boldsymbol{S} .
$$

Using Gauss's theorem, Poisson's equation $\nabla^{2} \Phi=4 \pi G \rho$, and the definition of the mass,

$$
L_{\mathrm{E}}=\frac{c}{\kappa} \int_{V} \nabla^{2} \Phi \mathrm{d} V=\frac{4 \pi G c}{\kappa} \int_{V} \rho \mathrm{d} V=\frac{4 \pi G M c}{\kappa}=1.3 \times 10^{44}\left(\frac{M_{\mathrm{BH}}}{10^{6} M_{\odot}}\right) \mathrm{erg} \mathrm{s}^{-1} .
$$

This sets the maximum luminosity of a source in hydrostatic equilibrium, $L_{\mathrm{Edd}}$ denotes Eddington luminosity. Note that we did not assume spherical geometry. While the effects of rotation can somewhat change this theoretical limit in an accretion disk, observed quasars for which black hole masses can be measured by independent methods appear to respect this limit.

The total luminosity from gas accreting onto a black hole, $L$, can be written as some radiative efficiency $\epsilon$ times the mass accretion rate $\dot{M}$,

$$
L=\epsilon \dot{M} c^{2}
$$

with the black hole accreting the non-radiated component, $\dot{M}_{\mathrm{BH}}=(1-\epsilon) \dot{M}$. The equation that governs the growth of the black hole mass is then

$$
\dot{M}_{\mathrm{BH}}=\frac{M_{\mathrm{BH}}}{t_{\mathrm{Edd}}},
$$

where (after substituting all fundamental constants),

$$
t_{\text {Edd }}=4 \times 10^{7}\left(\frac{\epsilon /(1-\epsilon)}{0.1}\right)\left(\frac{L}{L_{\mathrm{Edd}}}\right)^{-1} \mathrm{yr} .
$$

We therefore find that as long as fuel is amply supplied, the black hole mass grows exponentially in time, $M_{\mathrm{BH}} \propto \exp \left(t / t_{\mathrm{Edd}}\right)$, with an $e$-folding time $t_{\mathrm{Edd}}$. Since the growth time in equation (8) is significantly shorter than the $\sim 10^{9}$ years corresponding to the age of the Universe at a redshift $z \sim 6$ - where black holes with a mass $\sim 10^{9} M_{\odot}$ are found - one might naively conclude that there is plenty of time to grow the observed black hole masses from small seeds. For example, a seed black hole from a Population III star of $100 M_{\odot}$ can grow in less than a billion years up to $\sim 10^{9} M_{\odot}$ for $\epsilon \sim 0.1$ and $L \sim L_{\mathrm{Edd}}$. However, the intervention of various processes makes it unlikely that a stellar mass seed will be able to accrete continuously at its Eddington limit without interruption. 
Mergers are very common in the early Universe. Every time two gas-rich galaxies come together, their black holes are likely to coalesce. The coalescence is initially triggered by "dynamical friction" from the surrounding gas and stars (see Fig. 3.3, and is completed - when the binary gets tight - as a result of the emission of gravitational radiation (Begelman et al. 1980). Computer simulations reveal that when two black holes with unequal masses merge to make a single black hole, the remnant gets a kick due to the non-isotropic emission of gravitational radiation at the final plunge. This kick was calculated recently using advanced computer codes that solve Einstein's equations, a task that was plagued for decades with numerical instabilities Pretorius 2005; Campanelli et al. 2006; Baker et al. 2006). The typical kick velocity is hundreds of kilometer per second (and up to ten times more for special spin orientations), much larger than the escape speed from the first dwarf galaxies. This implies that continuous accretion was likely punctuated by black hole ejection events (Blecha and Loeb 2008; Tanaka and Haiman 2009), forcing the merged dwarf galaxy to grow a new black hole seed from scratch. These black hole recoils might have left observable signatures in the local Universe. For example, the halo of the Milky Way galaxy may include hundreds of freely-floating ejected black holes with compact star clusters around them, representing relics of the early mergers that assembled the Milky Way out of its original building blocks of dwarf galaxies (O'Learv and Loeb 2009).

\subsection{Seeds of Supermassive Black Holes}

Supermassive black holes with masses exceeding $10^{9} \mathrm{M}_{\odot}$ are observed at cosmological distances where the Universe was less than a billion years old. Assuming that their luminosity does not exceed the Eddington limit and the radiative efficiency is $\sim 10 \%$, one is driven to the conclusion that the black hole seeds must have started more massive than the stellar regime of $\lesssim 100 M_{\odot}$. The needed seeds may originate from supermassive stars, defined as hydrostatic configurations with masses $10^{3}-10^{8} M_{\odot}$ Loeb and Rasio 1994; Bromm and Larson 2004). Lacking carbon, nitrogen, oxygen, and iron, these first stars do not drive powerful stellar winds as present-day massive stars. Such systems have not been observed as of yet. Theoretically, they are expected to be supported almost entirely by radiation pressure and hence their luminosity equals the Eddington limit (equation 5). Supermassive stars steadily contract and convert their gravitational binding energy to radiation with a total lifetime $\lesssim 10^{6}$ yr before they collapse to a black hole.

Is it possible to make such stars in early galaxies? Numerical simulations indicate that stars of mass $\sim 10^{6} M_{\odot}$ could have formed at the centers of early dwarf galaxies that were barely able to cool their gas through transitions of atomic hydrogen, having $T_{\text {vir }} \sim 10^{4} \mathrm{~K}$ and no $\mathrm{H}_{2}$ molecules (which were suppressed by a Lyman-Werner background, Bromm and Loeb 2003; Diikstra et al. 2008; Regan and Haehnelt 2009). Such systems have a total mass that is several orders of magnitude higher than the earliest Jeans-mass condensations. In both cases, the gas lacks the ability to cool well below $T_{\text {vir }}$, and so it fragments into one or two major clumps. The simulation results in clumps of several million solar masses, which inevitably form massive black holes. The existence of such massive seeds would have given a jump start to the black hole growth process.

The nearly uniform entropy established by convection makes the structure of supermassive stars simple (equivalent to a so-called polytrope with an index $n=3$ ) with 
a unique relation between their central temperature $T_{c}$ and central density $\rho_{c}$,

$$
T_{c}=2 \times 10^{6} \mathrm{~K}\left(\frac{\rho_{c}}{1 \mathrm{~g} \mathrm{~cm}^{-3}}\right)^{1 / 3}\left(\frac{M_{\star}}{10^{6} M_{\odot}}\right)^{1 / 6} .
$$

Because of this modest temperature, nuclear reactions are insignificant in metal-poor stars with masses $M_{\star}>10^{5} M_{\odot}$. General relativistic corrections make the star unstable to direct collapse to a black hole as soon as its radius contracts to a value

$$
R_{\star}<R_{\text {crit }}=1.59 \times 10^{3}\left(\frac{M_{\star}}{10^{6} M_{\odot}}\right)^{1 / 2}\left(\frac{G M_{\star}}{c^{2}}\right) .
$$

Rotation can stabilize supermassive stars to smaller radii, but even rotating stars are expected to eventually collapse to a black hole after shedding their angular momentum through a wind (Saijo et al. 2002). If the supermassive star is made of pre-enriched gas, then powerful winds will inevitably be driven at its surface where the opacity due to lines from heavy elements far exceeds the Thomson value, making the outward radiation force stronger than gravity.

We note that the infall of a sufficiently dense, optically-thick spherical envelope of gas cannot be prevented by radiation pressure even if the radiation production rate exceeds the Eddington limit near the center. So long as the mass infall rate is sufficiently high, the Eddington limit will not apply because the photons will be trapped in the flow. Super-Eddington accretion can grow a seed black hole rapidly (as in the case of stellar collapse), as long as the blanket of infalling gas advects the radiation inwards as it accretes onto the black hole. This "obscured" mode of black hole accretion (which is hidden from view) could be particularly important at high redshifts when the gas density and infall rate onto galaxies obtain their highest values (Wyithe and Loeb 2012). We discuss the physics of this possibility in $\S 3$ below.

\subsection{Intermediate mass black holes}

Up to this point we have discussed the two well known classes of black holes: the stellar-mass and supermassive black holes. An intriguing question is whether there is an intermediate population of black holes in the mass range $M_{\mathrm{imbh}} \sim 100-10^{5} \mathrm{M}_{\odot}$ (Miller and Colbert 2004). These objects, referred to as intermediate mass black holes (IMBHs), are interesting for several reasons: they may represent seeds for forming SMBHs through accretion, they could stabilize globular clusters against core collapse (Baumgardt et al. 2004), they can lead to dark matter overdensities which will cause excessive dark matter annihilation signals (Bertone et al. 2005, 2009), they may have participated in cosmic reionization (Madau et al. 2004), and may provide sources of gravitational waves for direct detection (Wvithe and Loeb 2003, 2004; Mandel et al. 2008; O'Leary et al. 2009; Kocsis et al. 2012).

Several theoretical arguments have been put forth for forming IMBHs. The collapse of the first supermassive Pop-III stars may form IMBHs. This process can add $\sim 50\left(M_{\mathrm{imbh}} / 150 \mathrm{M}_{\odot}\right)$ IMBHs to galactic centers (Madau and Rees 2001). Secondly, runaway collisions of stars (Portegies Zwart and McMillan 2002; Freitag et al. 2006) or collisions with stellar black holes (O'Learv et al. 2006) in dense star clusters can also produce IMBHs. The clusters sink to the galactic nucleus due to dynamical friction $(\S 3.3)$ and deposit their IMBHs. This channel may supply an additional 50 IMBHs 
of mass $10^{3} \mathrm{M}_{\odot}$ within the inner $10 \mathrm{pc}$ (Portegies Zwart et al. 2006). IMBHs can also form and migrate inwards in the accretion disks of SMBHs (Goodman and Tan 2004; Levin 2007; Kocsis et al. 2011; McKernan et al. 2012).

Ultraluminous X-ray sources (ULXs) provide the best observational candidates for IMBHs (see $\S$ below). In particular, the estimated IMBH mass in ESO 243-49 HLX-1 based on its X-ray thermal accretion disk spectrum and radio signal is in the range $9 \times 10^{3}-9 \times 10^{4} \mathrm{M}_{\odot}$ (Davis et al. 2011; Webb et al. 2012). IMBHs may also represent low luminosity X-ray sources in the Galactic bulge accreting gas from the interstellar medium or infalling gas clouds (Bartos et al. 2013).

\section{Bondi-Hoyle-Lyttleton Accretion}

Bondi-Hoyle-Lyttleton accretion denotes a general class of black hole accretion, where the black hole is completely embedded in a gaseous medium and the inflow is spherical without a significant rotation. This occurs if black holes accrete from the ambient interstellar medium, as they pass through a dense gas cloud, become embedded in the envelope of a giant star, or if they accrete from a powerful wind of a binary companion star. Stellar mass black holes embedded in the accretion disk of a supermassive black hole may also accrete from the gaseous medium through this mode of accretion. Here we review the physical principles of Bondi-Hoyle-Lyttleton accretion and its observational implications.

\subsection{Simple model for spherical accretion}

Consider a black hole of mass $M$ is moving with relative velocity $V$ in an ambient medium of density $\rho_{0}$ and temperature $T_{0}$. The root-mean-square velocity of thermal protons in the gas relative to the black hole is roughly $v_{\mathrm{rms}}=\sqrt{c_{s}^{2}+V^{2}}$, where $c_{s} \approx \sqrt{\mathrm{k}_{\mathrm{B}} T / \mathrm{m}_{\mathrm{p}}}$ is the sound speed. The gas particles interior to the Bondi radius,

$$
r_{\mathrm{B}}=\frac{2 G M}{v_{\mathrm{rms}}^{2}}
$$

are gravitationally bound to the black hole and are accreted. The steady mass flux of particles entering this radius is $\rho_{0} v_{\mathrm{rms}}$. Multiplying this flux by the surface area associated with the Bondi radius, $\pi r_{\mathrm{B}}^{2}$, gives the supply rate of gas,

$$
\dot{M}_{\mathrm{B}}=4 \pi \rho_{0} \frac{G^{2} M^{2}}{v_{\mathrm{rms}}^{3}}=78\left(\frac{M}{10^{8} M_{\odot}}\right)^{2}\left(\frac{n_{H}}{1 \mathrm{~cm}^{-3}}\right)\left(\frac{T_{0}}{10^{4} \mathrm{~K}}\right)^{-3 / 2} M_{\odot} \mathrm{yr}^{-1} .
$$

where the second equality assumes a static medium $\left(v_{\mathrm{rms}}=c_{s}\right)$ and $n_{H}=\rho_{0} / \mathrm{m}_{\mathrm{p}}$. In a steady state this supply rate equals the mass accretion rate into the black hole.

This simple estimate for quasi-spherical accretion onto black holes is consistent with a range of simplified models. Hovle and Lyttleton (1939) derived the accretion onto a point mass in the limit that the gas pressure is negligible $\left(c_{s}=0\right)$ and particles move on ballistic orbits. Bondi and Hovle (1944) extended the analysis to include accretion from an axisymmetric wake behind the black hole, which led to a similar result, modified by a factor $\sim 1 / 2$. Bondi (1952) solved the Euler and continuity equations for a spherically symmetric steady-state adiabatic flow of gas assuming that the accreting object does 
not move relative to the medium $(V=0)$. Well inside the sonic radius (i.e., the point at which the infall and sound speeds are equal), the velocity is close to free-fall $v \sim$ $(2 G M / r)^{1 / 2}$ and the gas density is $\rho(r) \sim \rho_{0}\left(r / r_{\mathrm{B}}\right)^{-3 / 2}$. If the black hole moves with a supersonic velocity $V>c_{s}$, then a shock wave forms behind the hole, and the accretion occurs primarily from the shocked gas in a column trailing the black hole. The Bondi solution is then appropriate interior to the shock front and the Bondi radius $r_{B}$. The correction factor of this detailed calculation for the accretion rate relative to equation (12) is of order unity (see Ruffert 1994 for an updated formula).

\subsection{Luminosity}

The luminosity of an accretion flow is generally expressed in terms of the radiative efficiency $\epsilon$ for converting rest mass to radiation as, $L=\epsilon \dot{M} c^{2}$. The accretion rate for Bondi-Hoyle-Lyttleton accretion, $\dot{M}_{B}$, is given by equation (12). The radiative efficiency, $\epsilon$ is very small during spherical accretion since most of the energy, in the form of kinetic energy, heat, and radiation, is advected into the black hole rather than escape to infinity. This is due to the fact that the cooling time is longer than its accretion (free-fall) time or the diffusion time of the radiation outwards is much longer than the free-fall time and the radiation is trapped in the flow Rees 1978; Begelman 1979; Blondin 1986). The infall time of the fluid element from the Bondi radius to to the $\mathrm{BH}$ is approximately, $t_{\mathrm{ff}}(r)=r_{\mathrm{B}} / v_{\mathrm{rms}}$ and the photon diffusion time in the optically thick limit (i.e. where the optical depth satisfies $\tau=\kappa \rho r_{B} \gg 1$ ) is

$$
t_{\mathrm{diff}}(r)=\frac{1}{2 c} \int_{0}^{r_{\mathrm{B}}} \kappa \rho(\xi) \xi \mathrm{d} \xi=\frac{\kappa}{\pi c} \frac{\dot{M}_{\mathrm{B}}}{v_{\mathrm{rms}}}
$$

where we have used equations (11) and (12), $\rho(r) \propto r^{-3 / 2}$ for Bondi accretion, and $\kappa$ denotes the opacity. Thus, the diffusion time is larger than the infall time if

$$
\dot{M}_{\mathrm{B}} \gtrsim \frac{\pi r_{\mathrm{B}} c}{\kappa} \simeq \frac{2 \pi G M c}{\kappa v_{\mathrm{rms}}^{2}}=\frac{\dot{M}_{\mathrm{Edd}}}{2}\left(\frac{v_{\mathrm{rms}}}{c}\right)^{-2} .
$$

where in the last equality we used the definition of the Eddington luminosity, $L_{\text {Edd }}$ and the Eddington accretion rate $\dot{M}_{\mathrm{Edd}}=L_{\mathrm{Edd}} / c^{2}$. The radiation is trapped in the flow for hyper-Eddington accretion rates that satisfy equation (14). For smaller $\dot{M}_{\mathrm{B}}$, the inflowing gas can cool radiatively. Radiation-hydrodynamical simulations show that the flow remains radiatively inefficient, and the luminosity significantly sub-Eddington even if the accretion is super-Eddington. For $300 \geq \dot{m}_{\mathrm{B}} \equiv \dot{M}_{B} / \dot{M}_{\mathrm{Edd}} \gg 1$, the radiative efficiency is estimated to be in the range between $10^{-6} \lesssim \epsilon \lesssim 10^{-3}$ (Fragile et al. 2012) and $\epsilon \sim 10^{-2}$ (Roedig et al. 2012). If the accretion rate is very sub-Eddington, $\dot{m}_{\mathrm{B}} \ll 0.1$, the radiative efficiency is expected to follow $\epsilon=10 \dot{m}_{\mathrm{B}}$ (see $\S 4.2$ below).

\subsection{Dynamical Friction}

In addition to mass accretion, a gravitating object experiences a drag when moving through a medium. The drag arises due to the gravitational focusing of material forming a wake behind the accretion. This process in collisionless astronomical systems is called dynamical friction (Chandrasekhar 1943; Binney and Tremaine 2008). It is 
responsible for delivering massive black holes to the centers of galaxies after galaxy mergers (Begelman et al. 1980), sinking satellites in dark matter galaxy halos and thereby transporting stars and possibly intermediate mass black holes to the galactic nucleus (Portegies Zwart et al. 2006). A similar effect arises in a gaseous medium (Rephaeli and Salpeter 1980; Ostriker 1999). The steady-state drag force is

$$
F_{\mathrm{DF}}=-4 \pi \rho_{0} \frac{G M}{V^{2}} I
$$

where

$$
\begin{aligned}
I_{\text {supersonic }} & =\ln \left(\frac{r_{\max }}{r_{\min }}\right)+\frac{1}{2} \ln \left(1-\frac{1}{\mathcal{M}^{2}}\right) \text { if } \mathcal{M}>1, \\
I_{\text {subsonic }} & =\frac{1}{2} \ln \left(\frac{1+\mathcal{M}}{1-\mathcal{M}}\right)-\mathcal{M} \text { if } \mathcal{M}<1,
\end{aligned}
$$

and $\mathcal{M}=V / c_{s}$ is the Mach number, $r_{\max }$ denotes the maximum size of the medium and $r_{\text {min }}$ denotes the size of the perturber. For black holes $r_{\min }$ is of order the gravitational radius, $G M / c^{2}$. In the limit $\mathcal{M} \gg 1, F_{\mathrm{DF}} \approx-\dot{M}_{B} V \ln \left(r_{\max } / r_{\min }\right)$, and for $\mathcal{M} \ll 1$, $F_{\mathrm{DF}} \approx-\frac{1}{3} \dot{M}_{B} V$.

3.4 Vorticity, turbulence, and radiation effects

The simple model of Bondi-Hoyle-Lyttleton accretion neglects rotation, gas cooling, radiative effects, turbulence, and relativistic effects, which may modify the results significantly. These effects may be studied using state-of-the-art numerical simulations, which is becoming possible only recently.

Krumholz et al. (2005) examined Bondi accretion if the gas has a non-zero angular momentum beyond the Bondi radius. Neglecting radiation and thermodynamic effects, their simulation results are consistent with the fitting formula

$$
\dot{M}_{\text {vort }}=\dot{M}_{B} \times\left\{\begin{array}{lll}
0.4 & \text { if } & \omega_{*} \ll 1 \\
0.08 \omega_{*}^{-1} \ln \left(16 \omega_{*}\right) & \text { if } & \omega_{*} \gg 1
\end{array}\right.
$$

where $\omega_{*}=\omega r_{\mathrm{B}} /\left(2 c_{s}\right)$ is the vorticity parameter, where $\omega$ is the angular velocity of the inflowing gas at the outer boundary with respect to the black hole. This shows that the accretion rate is significantly suppressed by vorticity.

Further, Krumholz et al. (2006) have shown that the accretion rate is significantly modified in a turbulent medium over the estimate one obtains by using the turbulent velocity dispersion in equation (12). In this case the accretion follows a lognormal distribution with a median that is roughly given by

$$
\dot{M}_{\text {turb }}=\left[\dot{M}_{\mathrm{B}}^{-2}+\dot{M}_{\text {vort }}^{-2}\right]^{-1 / 2} \text {. }
$$

These estimates assumed that the velocity of the object is much smaller than the turbulent velocity dispersion.

Incorporating radiation and realistic heating and cooling processes between multiple components (electrons, ions, and photons) poses a challenge to realistic numerical simulations of Bondi-Hoyle-Lyttleton accretion. Recently, Fragile et al. (2012) have examined optically thick Bondi accretion using a general relativistic radiation magnetohydrodynamic (GR-R-MHD) simulation with $10 \leq \dot{M} / \dot{M}_{\text {Edd }} \leq 300$. They assumed two 
components: radiation and gas, with two gas-cooling mechanisms, Thomson scattering and thermal bremsstrahlung. The ambient gas temperature was between $10^{5}$ and $10^{7} \mathrm{~K}$. They found that the radiation pressure remained sub-dominant throughout the flow, and the luminosity remained very sub-Eddington despite the super-Eddington accretion rates such that the radiative efficiency was $\epsilon=L / \dot{M} c^{2} \lesssim 10^{-4}$. These simulations suggest that radiation does not have a major effect on the results. The opposite conclusion was reached based an independent code by Zanotti et al. (2011) and Roedig et al. (2012). These simulations also assumed two components (gas+radiation) with Thomson scattering and bremsstrahlung. In these simulations, radiation pressure dominated over gas pressure, and suppressed accretion by two orders of magnitude. The accretion rate was still super-Eddington in the simulation, while the luminosity was around the Eddington limit with $\epsilon \sim 10^{-2}$. These simulations showed that radiation pressure may serve to further stabilize the flow against a flip-flop instability (see $\S$ 3.5). While this progress is exciting, further improvements are necessary for a more secure estimate of the radiative efficiency of Bondi-Hoyle-Lyttleton accretion: primarily by incorporating the cooling effects of electrons (synchrotron emission and Comptonization) and extending calculations to smaller temperatures and higher resolution.

\subsection{Instabilities}

The Bondi-Hoyle-Lyttleton flow in not stable. Two dimensional numerical simulations have shown that the wake oscillates back and forth, exhibiting a "flip-flop instability" (Anzer et al. 1987; Fryxell and Taam 1988; Blondin and Pope 2009; Lora-Clavijo and Guzmán 2013), however, the structure of the wake is more regular in 3D simulations (Ruffert 1999). At very high Mach numbers, the accretion line behind the black hole is subject to a longitudinal (Cowie 1977) and transverse instabilities (Soker 1990, 1991). Furthermore, Nakayama (1994) has shown that an an axisymmetric shock is susceptible to a radial instability if the flow is accelerated after the shock. Ruffert (1999) investigated the Kelvin-Helmholtz and Rayleigh-Taylor instabilities, and concluded that these are not sufficient to explain the simulations without a feedback mechanism.

Bondi-Hoyle-Lyttleton accretion in a supersonic medium is subject to the standing accretion shock instability (SASI). The physical reason for this instability is the advective-acoustic cycle (Foglizzo 2002; Foglizzo et al. 2005; Guilet and Foglizzo 2012): entropy/vorticity perturbations are generated at the shock and advected to the sonic point, where an acoustic wave is excited and propagated back to the shock, leading to the growth of the entropy/vorticity perturbation. SASI helps drive supernova explosions (Burrows et al. 2006) and causes the emission of gravitational waves (Kotake et al. 2007).

Recent three dimensional radiation-hydrodynamical simulations revealed strong transient oscillations as the radiation pressure increases and becomes comparable to the gas pressure, eventually reversing the shock from the downstream to the upstream domain (Roedig et al. 2012). 


\section{Disk Accretion}

\subsection{Thin Disk Accretion}

If the inflow is endowed with rotation with respect to the black hole, it reaches a centrifugal barrier from where it cannot accrete farther inwards unless its angular momentum is transported away. Near the centrifugal barrier, where the gas is held against gravity by rotation, an accretion disk forms around the black hole, centered on the plane perpendicular to the rotation axis. The accretion time is dictated by the rate at which angular momentum is transported through viscous stress which could be significantly longer than the free-fall time for a non-rotating flow (as in the Bondi solution described in $\S 3$. In the absence of radiative processes, the dissipation of the gas's kinetic energy into heat would make the disk thick and hot, with a kinetic energy of the gas close to half the gravitational potential energy (virial equilibrium). For protons at distance $r$, this corresponds to a temperature $\sim 10^{13} \mathrm{~K}\left(r / r_{\mathrm{g}}\right)^{-1}$ where $r_{\mathrm{g}}=G M / c^{2}$ is the gravitational radius. However, if the cooling time of the gas is shorter than the viscous time, then a thin disk would form. This latter regime is realized for the high gas inflow rate during quasar activity $(\S 2.3)$, as well as for stellar mass black holes that emit in the soft thermal state in LMXBs ( $\S 2.2$. To better understand such objects, we start by exploring the structure of thin disks that characterize the high accretion rate of quasars (Shakura and Sunvaev 1973; Novikov and Thorne 1973).

We imagine a planar thin disk of cold gas orbiting a central black hole. Each gas element orbits at the local Keplerian velocity $v_{\phi}=r \Omega=(G M / r)^{1 / 2}$ and spirals slowly inwards with radial velocity $v_{r} \ll v_{\phi}$ as viscous torques transport its angular momentum to the outer part of the disk. The associated viscous stress generates heat, which is radiated away locally from the the disk surface. We assume that the disk is fed steadily and so it manifests a constant mass accretion rate at all radii. Mass conservation implies that

$$
\dot{M}=2 \pi r \Sigma v_{r}=\text { constant }
$$

independent of radius and time, where $\Sigma(r)$ is the surface mass density of the disk. The accretion rate $\dot{M}$ is a free parameter of the model, which is often expressed with the accretion rate corresponding to the Eddington luminosity as $\dot{m}=\dot{M} / \dot{M}_{\mathrm{E}}$. The corresponding angular momentum flux is $\dot{M} r^{2} \Omega+$ constant.

In the limit of a geometrically thin disk with a scale height $H \ll r$, the hydrodynamic equations decouple in the radial and vertical directions. In the radial direction, the Keplerian velocity profile introduces shear which dissipates heat as neighboring fluid elements rub against each other. This power provides a local radiative flux that leaves the system vertically from the top and bottom surfaces of the disk,

$$
F_{\mathrm{rad}}=\frac{3}{8 \pi} \dot{M} \Omega^{2}\left(1-\frac{r^{2} \Omega}{\left[r^{2} \Omega\right]_{\mathrm{ISCO}}}\right)=\frac{3}{8 \pi} \frac{G M \dot{M}}{r^{3}}\left[1-\left(\frac{r_{\mathrm{ISCO}}}{r}\right)^{1 / 2}\right] .
$$

where we assumed that the torque vanishes at the innermost stable circular orbit (ISCO), from where the gas plunges into the black hole over a free fall time. This sets the inner boundary of the disk. Here $r_{\mathrm{ISCO}}=6 r_{\mathrm{g}}$ for a non-spinning black hole, and $r_{\mathrm{g}} \leq r_{\mathrm{ISCO}} \leq 9 r_{\mathrm{g}}$ for a spinning black hole, where $r_{\mathrm{g}}=G M / c^{2}$. The total luminosity emitted by both faces of the disk is given by

$$
L=\int_{r_{\mathrm{ISCO}}}^{\infty} 2 F_{\mathrm{rad}} \times 2 \pi r \mathrm{~d} r=\frac{1}{2} \frac{G M \dot{M}}{r_{\mathrm{ISCO}}}=\frac{r_{g}}{2 r_{\mathrm{ISCO}}} \dot{M} c^{2},
$$


where we have ignored the radiation emitted inside the ISCO. This shows that the radiative efficiency of the disk is $\epsilon=r_{\mathrm{g}} /\left(2 r_{\mathrm{ISCO}}\right)=1 / 12=8.3 \%$ for a nonspinning black hole and $50 \%$ for a maximally spinning black hole in the prograde direction. Note that this simple estimate neglected general-relativistic corrections. A more detailed calculation gives similar results: $\epsilon=6 \%$ for nonspinning and $42 \%$ for maximally spinning black holes (Shapiro and Teukolskv 1983b).

In local thermodynamic equilibrium, the emergent flux from the surface of the disk (equation 21) can be written in terms of the disk surface temperature as $F_{\text {rad }}=\sigma_{\mathrm{SB}} T_{\mathrm{d}}^{4}$, where $\sigma_{\mathrm{SB}}$ is the Stephan-Boltzmann constant. This yields the following radial profile for the surface temperature of the disk,

$$
T_{\mathrm{d}}=\left(\frac{F_{\mathrm{rad}}}{\sigma_{\mathrm{SB}}}\right)^{1 / 4}=10^{5} \mathrm{~K}_{8}^{-1 / 4} \dot{m}_{-1}^{1 / 4} r_{1}^{-3 / 4}\left[1-\left(\frac{r}{r_{\mathrm{ISCO}}}\right)^{1 / 2}\right] .
$$

where $\dot{m}_{-1}=\dot{m} / 0.1, r_{1}=r /\left(10 r_{\mathrm{g}}\right)$, and $\dot{m}=\dot{M} / \dot{M}_{\text {Edd }}$. The corresponding thermal blackbody spectrum peaks in the UV bands for quasars, and in the X-ray band for stellar-mass black holes. (Non-thermal X-ray emission from a hot corona or a jet can supplement this disk emission.) Stellar-mass black holes can therefore be important $\mathrm{X}$-ray sources at high redshifts, especially if they are incorporated in a binary system where they accrete gas from a companion star (see $\S 2.2$.

Up to this point we did not need to adopt a model for the angular momentum transport (or an effective viscosity) in the disk. Indeed, the main observables, total luminosity and thermal spectrum, are very robustly set for a radiatively efficient thin disk by three parameters: $M, \dot{M}$, and the black hole spin which determines $r_{\text {ISCO }}$. Other details, involving the disk thickness, the radial surface density profile, and opacity however do depend on the model of viscosity and the relative contribution of radiation to gas pressure. The effective viscosity responsible is believed to be generated by the magneto-rotational instability (Balbus and Hawlev 1998). Assuming that (i) the angular momentum flux is proportional to the pressure in the disk with a dimensionless constant of proportionality $\alpha$, (ii) the disk is very thin so that it can be well approximated by vertically averaged quantities (one-zone model), (iii) the disk is optically thick in the vertical direction so that the gas and radiation are in thermal equilibrium, and (iv) the self-gravity of the gas is negligible, one can algebraically express all physical properties of the disk analytically (Goodman and Tan 2004). The surface density and mid-plane temperature are

$$
\begin{aligned}
\Sigma & =\frac{8\left(\mu \mathrm{m}_{\mathrm{p}} / \mathrm{k}_{\mathrm{B}}\right)^{4 / 5} \sigma_{\mathrm{SB}}^{1 / 5}}{3^{9 / 5} \alpha^{4 / 5} \kappa^{1 / 5}} \frac{\beta^{(1-b) 4 / 5}}{\Omega^{4 / 5}} F_{\mathrm{rad}}^{3 / 5}, \\
T_{\mathrm{c}} & =\frac{\left(\mu \mathrm{m}_{\mathrm{p}} / \mathrm{k}_{\mathrm{B}}\right)^{1 / 5} \kappa^{1 / 5}}{\left(3 \alpha \sigma_{\mathrm{SB}}\right)^{1 / 5}} \frac{\beta^{(1-b) / 5}}{\Omega^{1 / 5}} F_{\mathrm{rad}}^{2 / 5},
\end{aligned}
$$

where $\beta=p_{\text {gas }} /\left(p_{\text {gas }}+p_{\text {rad }}\right)$ is the gas to total pressure given by

$$
\frac{\beta^{(1 / 2)+(b-1) / 10}}{1-\beta}=\frac{c\left[k /\left(\mu \mathrm{m}_{\mathrm{p}}\right)\right]^{2 / 5}}{\left(3 \alpha \sigma_{\mathrm{SB}}\right)^{1 / 10} \kappa^{9 / 10}} \frac{\Omega^{9 / 10}}{F_{\mathrm{rad}}^{4 / 5}} .
$$

Here $b=0$ and 1 denote models where viscosity is proportional to the total pressure or only the gas pressure, respectively. Equations (24 26) along with equation (21) define the disk model as a function of radius. Equation (26) shows that in the inner regions 
$\beta \ll 1$, implying that radiation pressure dominates over gas pressure. In practice, radiation pressure dominates within $600 \alpha_{1}^{2 / 21} M_{5}^{2 / 21} r_{\mathrm{g}}$ if $\dot{M}=0.1 \dot{M}_{\text {Edd }}$ with $\epsilon=10 \%$ radiative efficiency. The opacity is dominated by electron scattering $\kappa_{\mathrm{es}}$ in the inner region and free-free absorption in the outer regions $\kappa_{\mathrm{ff}}$ (equation 3).

Vertical hydrostatic equilibrium implies $H=c_{s} / \Omega$, where $c_{s}=\sqrt{p / \rho}$ is the sound speed, where $\rho=\Sigma /(2 H)$ is the density, $p=p_{\text {gas }}+p_{\text {rad }}$ is the pressure, $p_{\text {gas }}=$ $\rho \mathrm{k}_{\mathrm{B}} T_{c} /\left(\mu \mathrm{m}_{\mathrm{p}}\right)$, and $p_{\mathrm{rad}}=\frac{1}{3} a_{\mathrm{rad}} T_{c}^{4}$. Here $\mu$ is the mean molecular weight, which is unity for a hydrogen plasma, and $\mu=0.615$ for $25 \%$ helium and $75 \%$ hydrogen by mass. Combining these equations, leads to $H=\kappa F_{\mathrm{rad}} /\left[c \Omega^{2}(1-\beta)\right] \sim(3 \kappa / 8 \pi c) \dot{M}(1-\beta)^{-1}$. When the accretion rate approaches the Eddington limit, $\beta \approx 0$, and the disk bloats and $H$ approaches $r$ in the inner regions, violating the thin-disk assumption. The model also breaks down if the accretion rate is several orders of magnitude below Eddington because the disk becomes optically thin $\tau=\frac{1}{2} \kappa \Sigma<1$.

The disk model described above ignores the self-gravity of the disk. This assumption is inevitably violated at large radii, where the disk becomes unstable to fragmentation due to its self-gravity, where the Toomre parameter, $Q=\left(c_{s} \Omega / \pi G \Sigma\right)$, drops below unity. Outside this radius, typically $\sim 2 \times 10^{4} r_{g}(\alpha / 0.1)^{0.6}\left(M / 10^{8} \mathrm{M}_{\odot}\right)^{1.2}$, the gas in quasar accretion disks would fragment into stars, and the stars may migrate inwards as the gas accretes onto the black hole. The energy output from stellar winds and supernovae would supplement the viscous heating of the disk and might regulate the disk to have $Q \sim 1$ outside the above boundary. We therefore conclude that star formation will inevitably occur on larger scales, before the gas is driven into the accretion disk that feeds the central black hole. Indeed, the broad emission lines of quasars display very high abundances of heavy elements in the spectra out to arbitrarily high redshifts. Since the total amount of mass in the disk interior to this radius makes only a small fraction of the mass of the supermassive black hole, quasars must be fed by gas that crosses this boundary after being vulnerable to fragmentation Milosavliević and Loeb 2004).

\subsection{Advection Dominated Accretion Flow}

When the accretion rate is considerably lower than the Eddington limit $\left(\dot{m} \lesssim 10^{-2}\right)$, the gas inflow switches to a different mode, called a Radiatively Inefficient Accretion Flow (RIAF) or an Advection Dominated Accretion Flow (ADAF), in which either the cooling time or the photon diffusion time is much longer than the accretion time of the gas and heat is mostly advected with the gas into the black hole (Naravan and Yi 1994). At the low gas densities and high temperatures characterizing this accretion mode, the Coulomb coupling is weak and the electrons do not heat up to the proton temperature even with the aid of plasma instabilities. Viscosity heats primarily the protons since they carry most of the momentum. The other major heat source, compression of the gas, also heats the protons more effectively than the electrons. As the gas falls inward and its density $\rho$ rises, the temperature of each species $T$ increases adiabatically as $T \propto \rho^{\gamma-1}$, where $\gamma$ is the corresponding adiabatic index. At radii $r \lesssim 10^{2} r_{\mathrm{Sch}}$, the electrons are relativistic with $\gamma=4 / 3$ and so their temperature rises inwards with increasing density as $T_{e} \propto \rho^{1 / 3}$ while the protons are non-relativistic with $\gamma=5 / 3$ and so $T_{\mathrm{p}} \propto \rho^{2 / 3}$, yielding a two-temperature plasma with the protons being much hotter than the electrons. Typical analytic models (Narayan and Yi 1994; Narayan and McClintock 2008, and references therein) yield $T_{p} \sim 10^{12} \mathrm{~K}\left(r / r_{\mathrm{Sch}}\right)^{-1}, T_{e} \sim \min \left(T_{p}, 10^{9-11} \mathrm{~K}\right)$. 
Since the typical sound speed is comparable to the Keplerian speed at each radius, the geometry of the flow is thick - making RIAFs the viscous analogs of Bondi accretions.

Analytic models imply a radial velocity that is a factor of $\sim \alpha$ smaller than the freefall speed and an accretion time that is a factor of $\sim \alpha$ longer than the free-fall time. However, since the sum of the kinetic and thermal energies of a proton is comparable to its gravitational binding energy, RIAFs are expected to be associated with strong outflows.

The radiative efficiency of RIAFs is smaller than the thin-disk value $(\epsilon)$. While the thin-disk value applies to high accretion rates above some critical value, $\dot{m}>\dot{m}_{\text {crit }}$, where $\dot{m}$ is the accretion rate in Eddington units, analytic RIAF models typically admit a radiative efficiency of

$$
\epsilon \approx 10 \dot{m} \text { if } \dot{m} \lesssim \dot{m}_{\text {crit }},
$$

with $\dot{m}_{\text {crit }} \sim 0.01$.

The nature of the flow can change when the disk becomes radiatively inefficient, since thermal convection may compete with MHD turbulence in transporting energy and angular momentum. In particular, the standard notion that viscosity is transported outwards in convective disks has been challenged in RIAFs (Naravan et al. 2000). In such convection-dominated disks the density profile is altered considerably from the standard ADAF models.

Since at low redshifts mergers are rare and much of the gas in galaxies has already been consumed in making stars, most local supermassive black holes are characterized by a very low accretion rate. The resulting low luminosity of these dormant black holes, such as the $4 \times 10^{6} M_{\odot}$ black hole lurking at the center of the Milky Way galaxy, is often described using RIAF/ADAF models. Although this mode of accretion is characterized by a low mass infall rate, it could persist over a period of time that is orders of magnitude longer than the quasar mode discussed earlier, so its contribution to the growth of black holes in galactic nuclei may not be negligible.

\subsection{Circumbinary SMBH disks}

Galaxy mergers naturally lead to SMBH binaries, which can also produce bright emission. Direct X-ray imaging of active nuclei have revealed several SMBH binaries at separations of $\sim 3 \mathrm{kpc}$ (Komossa et al. 2003; Ballo et al. 2004; Bianchi et al. 2008; Civano et al. 2012), 400 pc (Paggi et al. 2013), and 150 pc (Fabbiano et al. 2011). Additional evidence comes in the form of a radio galaxy with a double core with a projected separation of $10 \mathrm{pc}$ (Rodriguez et al. 2006), and several other observations of radio galaxies, such as the wiggled shape of jets indicating precession (Roos et al. 1993), the X-shaped morphologies of radio lobes (Merritt and Ekers 2002; Liu 2004), the interruption and recurrence of activity in double-double radio galaxies (Schoenmakers et al. 2000; Liu et al. 2003), and the elliptical motion of the unresolved core of 3C66B (Sudou et al. 2003), which have all been interpreted as indirect evidence for SMBH binaries down to subparsec scales. Velocity offsets in broad line spectra have also been observed in a handful of AGN which may be attributed to the orbital motion of the SMBH binary around the center of mass (Eracleous et al. 2012; Ju et al. 2013).

The structure of the accretion flow around close SMBH binaries is less understood. Generally speaking, the dense nuclear gas around the binary is expected to cool rapidly and settle in a rotationally supported circumbinary disk (Escala et al. 2005). If the 
binary plane is initially misaligned with the disk, the gravitational torques cause the disk to warp and twist. Viscosity then causes the disk to align with the binary on a timescale $t_{\mathrm{al}} \sim \max \left[\alpha(H / r)^{-2} t_{\text {orb }},(H / r)^{-1} t_{\text {orb }}\right]$ where $t_{\text {orb }}$ is the binary orbital period, $H$ is the scale-height, and $\alpha$ is the dimensionless viscosity parameter of a thin disk (Ivanov et al. 1999, and see $\S$ 4). For nearly equal masses, the accretion flow may then develop a triple-disk structure, forming individual accretion disks around the two binary components, and a larger circumbinary disk feeding these smaller disks (Hayasaki et al. 2008). For unequal mass binaries $m / M \ll 0.1$, the accretion disk geometry may be similar to a protoplanetary disk with a planet orbiting in the disk. The secondary excites spiral density waves in the disk which carry away energy and angular momentum from the secondary. This acts to reduce the density in a radial annulus of comparable to the Roche lobe $R_{R}$ (equation 11), regulates the inflow rate across the secondary orbit, and leads to the inward migration of the secondary. If the secondary is capable of quenching the inflow efficiently, a hollow cavity may form interior to its orbit with negligible accretion onto the primary. The gradual accretion from the outer regions will make the gas pile up outside the secondary's orbit similar to a river dam. The gas density and pressure increases significantly, until the gas overflows in non-axisymmetric streams, filling the cavity.

There are several interesting limiting cases for the circumbinary disk structure. For extreme binary mass ratios, $q \lesssim 10^{-4}$, the secondary cannot significantly modify the disk, which is then described by a standard thin disk assuming that the accretion rate is in the range $0.01 \dot{m}<0.3$ (see $\S$ 4). The spiral waves generated in the disk by the secondary carry away angular momentum very efficiently, shrinking the secondary's orbit quickly on the so-called Type- 1 migration timescale $\left(\sim 10^{4}-10^{5} \mathrm{yr}\right.$, Goldreich and Tremaine 1980). For comparable binary masses, $q \gtrsim 0.1$, a complete cavity forms, and the secondary is driven inwards on the viscous timescale $\left(\sim 10^{7} \mathrm{yr}\right)$ as the gas is flowing in from the outer regions (Type-2 migration). However, typically the local disk mass is much smaller than the secondary SMBH mass, implying that the gas piles up before it can drive the binary in. The accretion disk acquires a selfsimilar structure well outside the secondary as long as there is no inflow across the gap (Ivanov et al. 1999; Rafikov 2012). If the inflow into the cavity is significant, the disk settles in a steady state profile and the migration rate may be much slower (Type-1.5 migration, Kocsis et al. 2012b).

The accretion disk luminosity depends on whether the inner disk is truncated by tidal effects of the secondary and depends on the amount of gas pile up outside the secondary's orbit. The radiative efficiency may be larger than equation (22) due to the energy release corresponding to the inward migration of the secondary. This excess power comes from the part of the accretion disk outside the secondary's orbit where the gas pile up is significant. Figure 3 shows that this can make the disk significantly brighter in the optical bands for $q<0.01$ (Kocsis et al. 2012a). Furthermore, hydrodynamical simulations show that the accretion flow into the inner regions is expected to display periodic variability on roughly the orbital timescale. The variability power spectrum has discrete peaks, where the frequencies and harmonic weights depend on the binary mass ratio, accretion rate, and the $\alpha$ parameter (MacFadven and Milosavlievic 2008; Cuadra et al. 2009; Shi et al. 2012; Noble et al. 2012; (D'Orazio et al. 2012). However, further improvements are needed to include radiation pressure and extended to cover several viscous timescales to make more quantitative predictions on circumbinary accretion. 


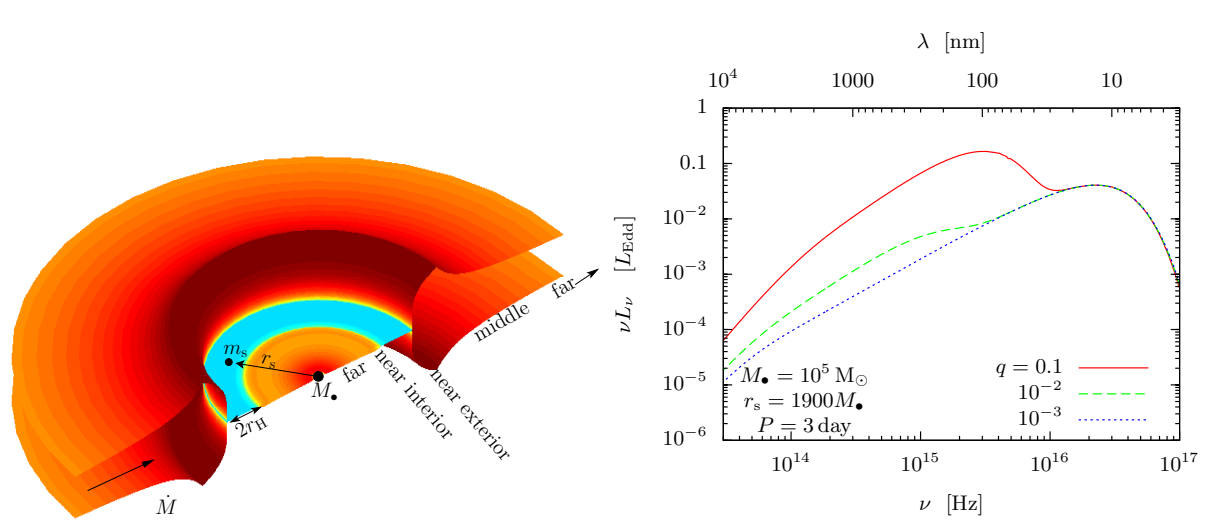

Fig. 3 Gas pile up due to a secondary in the accretion disk (left) and the corresponding thermal continuum spectrum for mass ratios $q=0.1,0.01$, and 0.001 (right). Figure credit: Kocsis et al. (2012a).

Supermassive black hole binaries may be identified in future surveys based on the velocity offsets in their broad line spectra (Shen and Loeb 2010; Eracleous et al. 2012), the expected periodic variability of the accretion luminosity on weeks-to-months timescales (Haiman et al. 2009), the excess brightness in the optical bands of the thermal spectrum corresponding to the pile up (Kocsis et al. 2012a), the variability of relativistic iron lines due to the orbital motion of the secondary and the effects of a gap in the disk (McKernan et al. 2013), or through the gravitational waves emitted by the binary (Kocsis et al. 2008; Lang et al. 2011).

\section{Feeding Black Holes with Stars}

\subsection{Tidal Disruption Events}

Most supermassive black holes in the local Universe are not accreting gas. They are typically lurking at the centers of galaxies, surrounded by a dense population of stars. A star that comes close to the black hole is torn apart by tidal gravity. The spaghettilike stellar debris falling back onto the black hole forms an accretion disk and produces a luminous electromagnetic transient lasting weeks to years, observable from cosmological distances (Frank and Rees 1976). Around 25 tidal disruption events have been observed to date in X-ray, UV, optical, and radio bands Komossa 2012; Gezari 2012, and references therein). These observations imply a stellar disruption rate of $3 \times 10^{-5} \mathrm{yr}^{-1}$ galaxy $^{-1}$ (van Velzen and Farrar 2012), consistent with theoretical rate estimates (Rees 1988; Wang and Merritt 2004).

The characteristic light-curve produced by tidal disruption events is derived as follows. The maximum distance at which the tidal field of the black hole is capable of unbinding a star is the tidal radius,

$$
R_{t}=\left(\frac{M_{\mathrm{BH}}}{M_{*}}\right)^{1 / 3} R_{*}=46 r_{\mathrm{g}}\left(\frac{M_{\mathrm{BH}}}{10^{6} \mathrm{M}_{\odot}}\right)^{-2 / 3}\left(\frac{M_{*}}{M_{\odot}}\right)^{-1 / 3} \frac{R_{*}}{R_{\odot}}
$$


The disruption occurs outside of the black hole horizon for $M_{\mathrm{BH}}<10^{8} \mathrm{M}_{\odot}$ for main sequence stars, $\lesssim 10^{6} \mathrm{M}_{\odot}$ for white dwarfs and $\lesssim 5 \mathrm{M}_{\odot}$ for neutron stars (see Kesden 2012 b, for highly spinning black holes where these bounds may be a factor 10 larger). After tidal disruption, approximately half of the stellar mass is bound to the black hole and half is unbound. The most bound material returns to pericenter after a time

$$
t_{\mathrm{fb}}=\frac{2 \pi}{6^{3 / 2}}\left(\frac{r_{p}}{R_{*}}\right)^{3 / 2} t_{p}=20\left(\frac{M_{\mathrm{BH}}}{10^{6} \mathrm{M}_{\odot}}\right)^{3 / 2}\left(\frac{r_{p}}{6 r_{g}}\right)^{3}\left(\frac{R_{*}}{\mathrm{R}_{\odot}}\right)^{-3 / 2} \min
$$

where $R_{p}$ is the pericenter distance, $t_{p}=\left(G M_{\mathrm{BH}} / R_{p}^{3}\right)^{-1 / 2}$ is the pericenter timescale. The fallback rate of bound material is then (Rees 1988)

$$
\dot{M}_{\mathrm{fb}}=\frac{\mathrm{d} M}{\mathrm{~d} E} \frac{\mathrm{d} E}{\mathrm{~d} t}=\frac{1}{3} \frac{M_{*}}{t_{\mathrm{fb}}}\left(\frac{t}{t_{\mathrm{fb}}}\right)^{-5 / 3}
$$

where $E \sim G M_{\mathrm{BH}} / a$ is the orbital energy, and $a \propto t^{-3 / 2}$ from Kepler's law, and we assumed that $\mathrm{d} M / \mathrm{d} E$ is constant. The timescale for the light-curve to reach the $t^{-5 / 3}$ limit depends on the stellar structure and spin through $\mathrm{d} M / \mathrm{d} E$ (Lodato et al. 2009; MacLeod et al. 2012; Guillochon and Ramirez-Ruiz 2013; Stone et al. 2012). This Newtonian estimate is modified for smaller stellar orbital eccentricities and by GR corrections, which depend on the spin of the black hole (Kesden 2012a; Havasaki et al. 2012; Dai et al. 2013).

Once the infalling gas returns to pericenter, it is expected to shock and form an accretion disk around the black hole. Initially, weeks to months after the disruption, the fallback rate is very super-Eddington. This phase is probably described by an advective slim disk with powerful outflows (Strubbe and Quataert 2009; De Colle et al. 2012; Tchekhovskoy et al. 2013). Months to a year later, the fallback rate becomes sub-Eddington, and the disk may radiatively cool efficiently to form a thin disk (§ 4). Finally, several years after the disruption, the fallback rate becomes very sub-Eddington $\dot{M}_{\mathrm{fb}} \lesssim 10^{-2} \dot{M}_{\mathrm{Edd}}$, and the disk becomes radiatively inefficient and hot $(\S 4.2)$ which may be accompanied by a jet. Two possible tidal disruption event sources have been observed to display jet activity (Swift J1644+57 and Swift J2058.4+0516, Burrows et al. 2011; Cenko et al. 2012). The lack of evidence for Lense-Thirring precession of the jet away from the observer suggests that the jet is aligned with the SMBH spin and not the accretion disk in these events (Stone and Loeb 2012a).

The bolometric luminosity of the system is $L=\epsilon \dot{M}_{\mathrm{fb}} c^{2} \propto t^{-5 / 3}$. In the thin disk phase, the multicolor blackbody spectrum of the accretion disk peaks in the soft X-ray bands. The light-curve in the X-ray bands follows $t^{-5 / 3}$ for several years, indeed consistent with many observed sources (Komossa 2012). Optical and UV wavelengths initially fall on the Rayleigh-Jeans tail of the disk spectrum, and the luminosity is initially proportional to the temperature $L_{\nu} \propto T_{\nu} \propto \dot{M}_{\mathrm{fb}}^{1 / 4} \propto t^{-5 / 12}$, and eventually converges to the $t^{-5 / 3}$ profile as the disk cools (Lodato and Rossi 2011). Such a shallower decline has been observed in the optical and UV bands for Swift J2058 (Cenko et al. 2012).

Upcoming surveys will detect more hundreds to thousands of tidal disruption events (TDEs). Multi-wavelength observations will enable us to probe the supermassive and intermediate-mass black hole populations, allowing us to measure their masses and spins (Kesden 2012a). Supermassive black hole binaries may imply a transient very high TDE rate of $1 \mathrm{yr}^{-1}$ and account overall for $10 \%$ of the cosmic TDE rate (Chen et al. 2011). Detecting multiple TDEs in the same galaxy within a few years time could 
be a signpost for a $\mathrm{MBH}$ binary. Merging $\mathrm{SMBH}$ receive a gravitational wave recoil kick due to anisotropic gravitational radiation. The recoiling black hole could produce tidal disruption flares spatially offset from the galactic center, which might contribute $1 \%$ of the TDE rates (Stone and Loeb 2012b). Finally, white dwarfs passing close to IMBHs may be detonated by the tidal gravity which would appear as underluminous supernovae (Carter and Luminet 1982; Rosswog et al. 2009; Haas et al. 2012).

\subsection{Fueling Active Galactic Nuclei Accretion Disks with Stars}

In the standard theory of AGN accretion, the effective viscosity responsible for delivering material onto the black hole is believed to be generated by the magneto-rotational instability ( $\S$ (4). An interesting alternative idea was examined by Goodman and Rafikov (2001) in which massive objects embedded in the accretion disk serve as an effective source of viscosity. These objects drive density waves in the disk which transport energy and angular momentum like a kinematic viscosity. In particular, stars in the stellar cluster around the supermassive black hole that cross the disk become captured in the disk due to accretion and hydrodynamical drag. Miralda-Escudé and Kollmeier (2005) have argued that the matter of these stars is sufficient to replenish the disk and fuel AGN. This process can also explain the observed correlation between the black hole mass and velocity dispersion of the stellar cluster ( $M-\sigma$ relation), although other explanations based on AGN feedback are also possible (see Chapter 5.6 in this book). Additionally, stars may also form in the outer regions of accretion disks due to gravitational fragmentation, and migrate inwards as they interact with the disk to fuel the AGN (Levin 2007).

The fate of the stars embedded in accretion disks is not well understood. Once they approach the black hole within the tidal radius (equation 28), they are tidally disrupted. However, their structure might change considerably before reaching this radius if they migrate across a gaseous disk. Accretion from the disk and mergers with other stars can lead to the formation of a supermassive star or an intermediate mass black hole already at large distances from the black hole (Goodman and Tan 2004; McKernan et al. 2012). If so, these objects orbiting around supermassive black holes will generate gravitational waves, and provide a sensitive new probe of the accretion disk (Kocsis et al. 2011).

\section{References}

U. Anzer, G. Boerner, J.J. Monaghan, Numerical studies of wind accretion. Astron. Astrophys. 176, 235-244 (1987)

J.G. Baker, J. Centrella, D.-I. Choi, M. Koppitz, J. van Meter, Gravitational-Wave Extraction from an Inspiraling Configuration of Merging Black Holes. Physical Review Letters 96(11), 111102 (2006). doi:10.1103/PhysRevLett.96.111102

S.A. Balbus, J.F. Hawley, Instability, turbulence, and enhanced transport in accretion disks. Reviews of Modern Physics 70, 1-53 (1998). doi:10.1103/RevModPhys.70.1

L. Ballo, V. Braito, R. Della Ceca, L. Maraschi, F. Tavecchio, M. Dadina, Arp 299: A Second Merging System with Two Active Nuclei? Astrophys. J. 600, 634-639 (2004). doi:10.1086/379887

I. Bartos, Z. Haiman, B. Kocsis, S. Marka, G2 can Illuminate the Black Hole Population near the Galactic Center. ArXiv e-prints (2013)

H. Baumgardt, J. Makino, T. Ebisuzaki, Massive Black Holes in Star Clusters. II. Realistic Cluster Models. Astrophys. J. 613, 1143-1156 (2004). doi:10.1086/423299 
M.C. Begelman, Can a spherically accreting black hole radiate very near the Eddington limit. Mon. Not. Roy. Acad. Soc. 187, 237-251 (1979)

M.C. Begelman, R.D. Blandford, M.J. Rees, Massive black hole binaries in active galactic nuclei. Nature 287, 307-309 (1980). doi:10.1038/287307a0

K. Belczynski, T. Bulik, C.L. Fryer, A. Ruiter, F. Valsecchi, J.S. Vink, J.R. Hurley, On the Maximum Mass of Stellar Black Holes. Astrophys. J. 714, 1217-1226 (2010). doi:10.1088/0004-637X/714/2/1217

G. Bertone, A.R. Zentner, J. Silk, New signature of dark matter annihilations: Gamma rays from intermediate-mass black holes. Phys. Rev. D 72(10), 103517 (2005). doi:10.1103/PhysRevD.72.103517

G. Bertone, M. Fornasa, M. Taoso, A.R. Zentner, Dark matter annihilation around intermediate mass black holes: an update. New Journal of Physics 11(10), 105016 (2009). doi:10.1088/1367-2630/11/10/105016

S. Bianchi, M. Chiaberge, E. Piconcelli, M. Guainazzi, G. Matt, Chandra unveils a binary active galactic nucleus in Mrk 463. Mon. Not. Roy. Acad. Soc. 386, 105-110 (2008). doi:10.1111/j.1365-2966.2008.13078.x

J. Binney, S. Tremaine, Galactic Dynamics: Second Edition (Princeton University Press, ???, 2008)

L. Blecha, A. Loeb, Effects of gravitational-wave recoil on the dynamics and growth of supermassive black holes. Mon. Not. Roy. Acad. Soc. 390, 1311-1325 (2008). doi:10.1111/j.13652966.2008.13790.x

J.M. Blondin, Hypercritical spherical accretion onto compact objects. Astrophys. J. 308, 755764 (1986). doi:10.1086/164548

J.M. Blondin, T.C. Pope, Revisiting the "Flip-Flop" Instability of Hoyle-Lyttleton Accretion. Astrophys. J. 700, 95-102 (2009). doi:10.1088/0004-637X/700/1/95

H. Bondi, On spherically symmetrical accretion. Mon. Not. Roy. Acad. Soc. 112, 195 (1952)

H. Bondi, F. Hoyle, On the mechanism of accretion by stars. Mon. Not. Roy. Acad. Soc. 104, $273(1944)$

V. Bromm, R.B. Larson, The First Stars. ARA\&A 42, $79-118$ (2004). doi:10.1146/annurev.astro.42.053102.134034

V. Bromm, A. Loeb, Formation of the First Supermassive Black Holes. Astrophys. J. 596, 34-46 (2003). doi:10.1086/377529

A. Burrows, E. Livne, L. Dessart, C.D. Ott, J. Murphy, A New Mechanism for Core-Collapse Supernova Explosions. Astrophys. J. 640, 878-890 (2006). doi:10.1086/500174

D.N. Burrows, J.A. Kennea, G. Ghisellini, V. Mangano, B. Zhang, K.L. Page, M. Eracleous, P. Romano, T. Sakamoto, A.D. Falcone, J.P. Osborne, S. Campana, A.P. Beardmore, A.A. Breeveld, M.M. Chester, R. Corbet, S. Covino, J.R. Cummings, P. D'Avanzo, V. D'Elia, P. Esposito, P.A. Evans, D. Fugazza, J.M. Gelbord, K. Hiroi, S.T. Holland, K.Y. Huang, M. Im, G. Israel, Y. Jeon, Y.-B. Jeon, H.D. Jun, N. Kawai, J.H. Kim, H.A. Krimm, F.E. Marshall, P. Mészáros, H. Negoro, N. Omodei, W.-K. Park, J.S. Perkins, M. Sugizaki, H.-I. Sung, G. Tagliaferri, E. Troja, Y. Ueda, Y. Urata, R. Usui, L.A. Antonelli, S.D. Barthelmy, G. Cusumano, P. Giommi, A. Melandri, M. Perri, J.L. Racusin, B. Sbarufatti, M.H. Siegel, N. Gehrels, Relativistic jet activity from the tidal disruption of a star by a massive black hole. Nature 476, 421-424 (2011). doi:10.1038/nature10374

M. Campanelli, C.O. Lousto, P. Marronetti, Y. Zlochower, Accurate Evolutions of Orbiting Black-Hole Binaries without Excision. Physical Review Letters 96(11), 111101 (2006). doi:10.1103/PhysRevLett.96.111101

B. Carter, J.P. Luminet, Pancake detonation of stars by black holes in galactic nuclei. Nature 296, 211-214 (1982). doi:10.1038/296211a0

S.B. Cenko, H.A. Krimm, A. Horesh, A. Rau, D.A. Frail, J.A. Kennea, A.J. Levan, S.T. Holland, N.R. Butler, R.M. Quimby, J.S. Bloom, A.V. Filippenko, A. Gal-Yam, J. Greiner, S.R. Kulkarni, E.O. Ofek, F. Olivares E., P. Schady, J.M. Silverman, N.R. Tanvir, D. Xu, Swift J2058.4+0516: Discovery of a Possible Second Relativistic Tidal Disruption Flare? Astrophys. J. 753, 77 (2012). doi:10.1088/0004-637X/753/1/77

S. Chandrasekhar, Dynamical Friction. I. General Considerations: the Coefficient of Dynamical Friction. Astrophys. J. 97, 255 (1943). doi:10.1086/144517

X. Chen, A. Sesana, P. Madau, F.K. Liu, Tidal Stellar Disruptions by Massive Black Hole Pairs. II. Decaying Binaries. Astrophys. J. 729, 13 (2011). doi:10.1088/0004-637X/729/1/13

F. Civano, M. Elvis, G. Lanzuisi, T. Aldcroft, M. Trichas, A. Bongiorno, M. Brusa, L. Blecha, A. Comastri, A. Loeb, M. Salvato, A. Fruscione, A. Koekemoer, S. Komossa, R. Gilli, V. 
Mainieri, E. Piconcelli, C. Vignali, Chandra High-resolution observations of CID-42, a Candidate Recoiling Supermassive Black Hole. Astrophys. J. 752, 49 (2012). doi:10.1088/0004$637 \mathrm{X} / 752 / 1 / 49$

L.L. Cowie, An investigation of the stability of the Bondi-Hoyle model of accretion flow. Mon. Not. Roy. Acad. Soc. 180, 491-494 (1977)

J. Cuadra, P.J. Armitage, R.D. Alexander, M.C. Begelman, Massive black hole binary mergers within subparsec scale gas discs. Mon. Not. Roy. Acad. Soc. 393, 1423-1432 (2009). doi:10.1111/j.1365-2966.2008.14147.x

L. Dai, A. Escala, P. Coppi, The Impact of Bound Stellar Orbits and General Relativity on the Temporal Behavior of Tidal Disruption Flares. ArXiv e-prints (2013)

S.W. Davis, R. Narayan, Y. Zhu, D. Barret, S.A. Farrell, O. Godet, M. Servillat, N.A. Webb, The Cool Accretion Disk in ESO 243-49 HLX-1: Further Evidence of an Intermediate-mass Black Hole. Astrophys. J. 734, 111 (2011). doi:10.1088/0004-637X/734/2/111

F. De Colle, J. Guillochon, J. Naiman, E. Ramirez-Ruiz, The Dynamics, Appearance, and Demographics of Relativistic Jets Triggered by Tidal Disruption of Stars in Quiescent Supermassive Black Holes. Astrophys. J. 760, 103 (2012). doi:10.1088/0004-637X/760/2/103

C.D. Dermer, A. Atoyan, Collapse of Neutron Stars to Black Holes in Binary Systems: A Model for Short Gamma-Ray Bursts. Astrophys. J. Lett. 643, 13-16 (2006). doi:10.1086/504895

M. Dijkstra, Z. Haiman, A. Mesinger, J.S.B. Wyithe, Fluctuations in the high-redshift LymanWerner background: close halo pairs as the origin of supermassive black holes. Mon. Not. Roy. Acad. Soc. 391, 1961-1972 (2008). doi:10.1111/j.1365-2966.2008.14031.x

D.J. D'Orazio, Z. Haiman, A. MacFadyen, Accretion into the Central Cavity of a Circumbinary Disk. ArXiv e-prints (2012)

M. Eracleous, T.A. Boroson, J.P. Halpern, J. Liu, A Large Systematic Search for Close Supermassive Binary and Rapidly Recoiling Black Holes. Astrophys. J. Suppl. 201, 23 (2012). doi:10.1088/0067-0049/201/2/23

A. Escala, R.B. Larson, P.S. Coppi, D. Mardones, The Role of Gas in the Merging of Massive Black Holes in Galactic Nuclei. II. Black Hole Merging in a Nuclear Gas Disk. Astrophys. J. 630, 152-166 (2005). doi:10.1086/431747

G. Fabbiano, J. Wang, M. Elvis, G. Risaliti, A close nuclear black-hole pair in the spiral galaxy NGC3393. Nature 477, 431-434 (2011). doi:10.1038/nature10364

R. Fernández, B.D. Metzger, Nuclear Dominated Accretion Flows in Two Dimensions. I. Torus Evolution with Parametric Microphysics. Astrophys. J. 763, 108 (2013). doi:10.1088/0004$637 \mathrm{X} / 763 / 2 / 108$

T. Foglizzo, Non-radial instabilities of isothermal Bondi accretion with a shock: Vorticalacoustic cycle vs. post-shock acceleration. Astron. Astrophys. 392, 353-368 (2002). doi:10.1051/0004-6361:20020912

T. Foglizzo, P. Galletti, M. Ruffert, A fresh look at the unstable simulations of BondiHoyle-Lyttleton accretion. Astron. Astrophys. 435, 397-411 (2005). doi:10.1051/00046361:20042201

P.C. Fragile, A. Gillespie, T. Monahan, M. Rodriguez, P. Anninos, Numerical Simulations of Optically Thick Accretion onto a Black Hole. I. Spherical Case. Astrophys. J. Suppl. 201, 9 (2012). doi:10.1088/0067-0049/201/2/9

J. Frank, M.J. Rees, Effects of massive central black holes on dense stellar systems. Mon. Not. Roy. Acad. Soc. 176, 633-647 (1976)

M. Freitag, M.A. Gürkan, F.A. Rasio, Runaway collisions in young star clusters - II. Numerical results. Mon. Not. Roy. Acad. Soc. 368, 141-161 (2006). doi:10.1111/j.13652966.2006.10096.x

C.L. Fryer, Mass Limits For Black Hole Formation. Astrophys. J. 522, 413-418 (1999). doi:10.1086/307647

B.A. Fryxell, R.E. Taam, Numerical simulation of nonaxisymmetric adiabatic accretion flow. Astrophys. J. 335, 862-880 (1988). doi:10.1086/166973

S. Gezari, Ultraviolet and Optical Observations of Tidal Disruption Events, in European Physical Journal Web of Conferences. European Physical Journal Web of Conferences, vol. 39, 2012, p. 3001. doi:10.1051/epjconf/20123903001

P. Goldreich, S. Tremaine, Disk-satellite interactions. Astrophys. J. 241, 425-441 (1980). doi:10.1086/158356

J. Goodman, R.R. Rafikov, Planetary Torques as the Viscosity of Protoplanetary Disks. Astrophys. J. 552, 793-802 (2001). doi:10.1086/320572

J. Goodman, J.C. Tan, Supermassive Stars in Quasar Disks. Astrophys. J. 608, 108-118 (2004). 
doi:10.1086/386360

H.-J. Grimm, M. Gilfanov, R. Sunyaev, High-mass X-ray binaries as a star formation rate indicator in distant galaxies. Mon. Not. Roy. Acad. Soc. 339, 793-809 (2003). doi:10.1046/j.1365-8711.2003.06224.x

J. Guilet, T. Foglizzo, On the linear growth mechanism driving the standing accretion shock instability. Mon. Not. Roy. Acad. Soc. 421, 546-560 (2012). doi:10.1111/j.13652966.2012.20333.x

J. Guillochon, E. Ramirez-Ruiz, Hydrodynamical Simulations to Determine the Feeding Rate of Black Holes by the Tidal Disruption of Stars: The Importance of the Impact Parameter and Stellar Structure. Astrophys. J. 767, 25 (2013). doi:10.1088/0004-637X/767/1/25

R. Haas, R.V. Shcherbakov, T. Bode, P. Laguna, Tidal Disruptions of White Dwarfs from Ultra-close Encounters with Intermediate-mass Spinning Black Holes. Astrophys. J. 749, 117 (2012). doi:10.1088/0004-637X/749/2/117

Z. Haiman, B. Kocsis, K. Menou, The Population of Viscosity- and Gravitational Wave-driven Supermassive Black Hole Binaries Among Luminous Active Galactic Nuclei. Astrophys. J. 700, 1952-1969 (2009). doi:10.1088/0004-637X/700/2/1952

K. Hayasaki, S. Mineshige, L.C. Ho, A Supermassive Binary Black Hole with Triple Disks. Astrophys. J. 682, 1134-1140 (2008). doi:10.1086/588837

K. Hayasaki, N. Stone, A. Loeb, Finite, Intense Accretion Bursts from Tidal Disruption of Stars on Bound Orbits. ArXiv e-prints (2012)

A. Heger, C.L. Fryer, S.E. Woosley, N. Langer, D.H. Hartmann, How Massive Single Stars End Their Life. Astrophys. J. 591, 288-300 (2003). doi:10.1086/375341

F. Hoyle, R.A. Lyttleton, The effect of interstellar matter on climatic variation. Proceedings of the Cambridge Philosophical Society 35, 405 (1939). doi:10.1017/S0305004100021150

P.B. Ivanov, J.C.B. Papaloizou, A.G. Polnarev, The evolution of a supermassive binary caused by an accretion disc. Mon. Not. Roy. Acad. Soc. 307, 79-90 (1999). doi:10.1046/j.13658711.1999.02623.x

W. Ju, J.E. Greene, R.R. Rafikov, S.J. Bickerton, C. Badenes, Search for Supermassive Black Hole Binaries in the Sloan Digital Sky Survey Spectroscopic Sample. ArXiv e-prints (2013)

M. Kesden, Black-hole spin dependence in the light curves of tidal disruption events. Phys. Rev. D 86(6), 064026 (2012a). doi:10.1103/PhysRevD.86.064026

M. Kesden, Tidal-disruption rate of stars by spinning supermassive black holes. Phys. Rev. D 85(2), 024037 (2012b). doi:10.1103/PhysRevD.85.024037

W. Kley, A. Shankar, A. Burkert, Radiation hydrodynamics of axisymmetric accretion flow in a common envelope environment. Astron. Astrophys. 297, 739 (1995)

B. Kocsis, Z. Haiman, A. Loeb, Gas pile-up, gap overflow and Type 1.5 migration in circumbinary discs: application to supermassive black hole binaries. Mon. Not. Roy. Acad. Soc. 427, 2680-2700 (2012a). doi:10.1111/j.1365-2966.2012.22118.x

B. Kocsis, Z. Haiman, A. Loeb, Gas pile-up, gap overflow and Type 1.5 migration in circumbinary discs: general theory. Mon. Not. Roy. Acad. Soc. 427, 2660-2679 (2012b). doi:10.1111/j.1365-2966.2012.22129.x

B. Kocsis, Z. Haiman, K. Menou, Premerger Localization of Gravitational Wave Standard Sirens with LISA: Triggered Search for an Electromagnetic Counterpart. Astrophys. J. 684, 870-887 (2008). doi:10.1086/590230

B. Kocsis, A. Ray, S. Portegies Zwart, Mapping the Galactic Center with Gravitational Wave Measurements Using Pulsar Timing. Astrophys. J. 752, 67 (2012). doi:10.1088/0004$637 \mathrm{X} / 752 / 1 / 67$

B. Kocsis, N. Yunes, A. Loeb, Observable signatures of extreme mass-ratio inspiral black hole binaries embedded in thin accretion disks. Phys. Rev. D 84(2), 024032 (2011). doi:10.1103/PhysRevD.84.024032

S. Komossa, Tidal Disruption of Stars by Supermassive Black Holes: The X-ray View, in European Physical Journal Web of Conferences. European Physical Journal Web of Conferences, vol. 39, 2012, p. 2001. doi:10.1051/epjconf/20123902001

S. Komossa, V. Burwitz, G. Hasinger, P. Predehl, J.S. Kaastra, Y. Ikebe, Discovery of a Binary Active Galactic Nucleus in the Ultraluminous Infrared Galaxy NGC 6240 Using Chandra. Astrophys. J. Lett. 582, 15-19 (2003). doi:10.1086/346145

K. Kotake, N. Ohnishi, S. Yamada, Gravitational Radiation from Standing Accretion Shock Instability in Core-Collapse Supernovae. Astrophys. J. 655, 406-415 (2007). doi:10.1086/509320

M.R. Krumholz, C.F. McKee, R.I. Klein, Bondi Accretion in the Presence of Vorticity. Astro- 
phys. J. 618, 757-768 (2005). doi:10.1086/426051

M.R. Krumholz, C.F. McKee, R.I. Klein, Bondi-Hoyle Accretion in a Turbulent Medium. Astrophys. J. 638, 369-381 (2006). doi:10.1086/498844

R.N. Lang, S.A. Hughes, N.J. Cornish, Measuring parameters of massive black hole binaries with partially aligned spins. Phys. Rev. D 84(2), 022002 (2011). doi:10.1103/PhysRevD.84.022002

Y. Levin, Starbursts near supermassive black holes: young stars in the Galactic Centre, and gravitational waves in LISA band. Mon. Not. Roy. Acad. Soc. 374, 515-524 (2007). doi:10.1111/j.1365-2966.2006.11155.x

J. Li, J. Ostriker, R. Sunyaev, Rotating Accretion Flows: From Infinity to the Black Hole. ArXiv e-prints (2012)

F.K. Liu, X-shaped radio galaxies as observational evidence for the interaction of supermassive binary black holes and accretion disc at parsec scale. Mon. Not. Roy. Acad. Soc. 347, 13571369 (2004). doi:10.1111/j.1365-2966.2004.073

F.K. Liu, X.-B. Wu, S.L. Cao, Double-double radio galaxies: remnants of merged supermassive binary black holes. Mon. Not. Roy. Acad. Soc. 340, 411-416 (2003). doi:10.1046/j.13658711.2003.06235.x

G. Lodato, E.M. Rossi, Multiband light curves of tidal disruption events. Mon. Not. Roy. Acad. Soc. 410, 359-367 (2011). doi:10.1111/j.1365-2966.2010.17448.x

G. Lodato, A.R. King, J.E. Pringle, Stellar disruption by a supermassive black hole: is the light curve really proportional to $t^{-5 / 3}$ ? Mon. Not. Roy. Acad. Soc. 392, 332-340 (2009). doi:10.1111/j.1365-2966.2008.14049.x

A. Loeb, F.A. Rasio, Collapse of primordial gas clouds and the formation of quasar black holes. Astrophys. J. 432, 52-61 (1994). doi:10.1086/174548

F.D. Lora-Clavijo, F.S. Guzmán, Axisymmetric Bondi-Hoyle accretion on to a Schwarzschild black hole: shock cone vibrations. Mon. Not. Roy. Acad. Soc. 429, 3144-3154 (2013). doi: $10.1093 / \mathrm{mnras} / \mathrm{sts} 573$

A.I. MacFadyen, M. Milosavljević, An Eccentric Circumbinary Accretion Disk and the Detection of Binary Massive Black Holes. Astrophys. J. 672, 83-93 (2008). doi:10.1086/523869

M. MacLeod, J. Guillochon, E. Ramirez-Ruiz, The Tidal Disruption of Giant Stars and their Contribution to the Flaring Supermassive Black Hole Population. Astrophys. J. 757, 134 (2012). doi:10.1088/0004-637X/757/2/134

P. Madau, M.J. Rees, Massive Black Holes as Population III Remnants. Astrophys. J. Lett. 551, 27-30 (2001). doi:10.1086/319848

P. Madau, M.J. Rees, M. Volonteri, F. Haardt, S.P. Oh, Early Reionization by Miniquasars. Astrophys. J. 604, 484-494 (2004). doi:10.1086/381935

I. Mandel, D.A. Brown, J.R. Gair, M.C. Miller, Rates and Characteristics of Intermediate Mass Ratio Inspirals Detectable by Advanced LIGO. Astrophys. J. 681, 1431-1447 (2008). doi:10.1086/588246

B. McKernan, K.E.S. Ford, W. Lyra, H.B. Perets, Intermediate mass black holes in AGN discs - I. Production and growth. Mon. Not. Roy. Acad. Soc. 425, 460-469 (2012). doi:10.1111/j.1365-2966.2012.21486.x

B. McKernan, K.E.S. Ford, B. Kocsis, Z. Haiman, Intermediate mass black holes in AGN discs - I. Production and growth. Mon. Not. Roy. Acad. Soc.in press (2013). doi:10.1111/j.13652966.2012.21486.x

D. Merritt, R.D. Ekers, Tracing Black Hole Mergers Through Radio Lobe Morphology. Science 297, 1310-1313 (2002). doi:10.1126/science.1074688

M.C. Miller, E.J.M. Colbert, Intermediate-Mass Black Holes. International Journal of Modern Physics D 13, 1-64 (2004). doi:10.1142/S0218271804004426

M. Milosavljević, A. Loeb, The Link between Warm Molecular Disks in Maser Nuclei and Star Formation near the Black Hole at the Galactic Center. Astrophys. J. Lett. 604, 45-48 (2004). doi: $10.1086 / 383467$

S. Mineo, M. Gilfanov, R. Sunyaev, X-ray emission from star-forming galaxies - I. High-mass X-ray binaries. Mon. Not. Roy. Acad. Soc. 419, 2095-2115 (2012). doi:10.1111/j.13652966.2011.19862.x

J. Miralda-Escudé, J.A. Kollmeier, Star Captures by Quasar Accretion Disks: A Possible Explanation of the M- $\sigma$ Relation. Astrophys. J. 619, 30-40 (2005). doi:10.1086/426467

M.P. Muno, J.S. Clark, P.A. Crowther, S.M. Dougherty, R. de Grijs, C. Law, S.L.W. McMillan, M.R. Morris, I. Negueruela, D. Pooley, S. Portegies Zwart, F. Yusef-Zadeh, A Neutron Star with a Massive Progenitor in Westerlund 1. Astrophys. J. Lett. 636, 41-44 (2006). 
doi:10.1086/499776

K. Nakayama, Dynamical Instability of Standing Shock Waves in Adiabatic Accretion Flows and Wind Flows. Mon. Not. Roy. Acad. Soc. 270, 871 (1994)

R. Narayan, J.E. McClintock, Advection-dominated accretion and the black hole event horizon. New A Rev. 51, 733-751 (2008). doi:10.1016/j.newar.2008.03.002

R. Narayan, I. Yi, Advection-dominated accretion: A self-similar solution. Astrophys. J. Lett. 428, 13-16 (1994). doi:10.1086/187381

R. Narayan, I.V. Igumenshchev, M.A. Abramowicz, Self-similar Accretion Flows with Convection. Astrophys. J. 539, 798-808 (2000). doi:10.1086/309268

S.C. Noble, B.C. Mundim, H. Nakano, J.H. Krolik, M. Campanelli, Y. Zlochower, N. Yunes, Circumbinary Magnetohydrodynamic Accretion into Inspiraling Binary Black Holes. Astrophys. J. 755, 51 (2012). doi:10.1088/0004-637X/755/1/51

I.D. Novikov, K.S. Thorne, Astrophysics of Black Holes., in Black Holes (Les Astres Occlus), ed. by A. Giannaras, 1973, pp. 343-450

R.M. O'Leary, A. Loeb, Star clusters around recoiled black holes in the Milky Way halo. Mon. Not. Roy. Acad. Soc. 395, 781-786 (2009). doi:10.1111/j.1365-2966.2009.14611.x

R.M. O'Leary, B. Kocsis, A. Loeb, Gravitational waves from scattering of stellar-mass black holes in galactic nuclei. Mon. Not. Roy. Acad. Soc. 395, 2127-2146 (2009). doi:10.1111/j.1365-2966.2009.14653.x

R.M. O'Leary, F.A. Rasio, J.M. Fregeau, N. Ivanova, R. O'Shaughnessy, Binary Mergers and Growth of Black Holes in Dense Star Clusters. Astrophys. J. 637, 937-951 (2006). doi:10.1086/498446

E.C. Ostriker, Dynamical Friction in a Gaseous Medium. Astrophys. J. 513, 252-258 (1999). doi:10.1086/306858

A. Paggi, G. Fabbiano, G. Risaliti, J. Wang, M. Elvis, Two Compton-Thick Active Nuclei in Arp 220? ArXiv e-prints (2013)

V. Paschalidis, M. MacLeod, T.W. Baumgarte, S.L. Shapiro, Merger of white dwarf-neutron star binaries: Prelude to hydrodynamic simulations in general relativity. Phys. Rev. D 80(2), 024006 (2009). doi:10.1103/PhysRevD.80.024006

S.F. Portegies Zwart, S.L.W. McMillan, The Runaway Growth of Intermediate-Mass Black Holes in Dense Star Clusters. Astrophys. J. 576, 899-907 (2002). doi:10.1086/341798

S.F. Portegies Zwart, H. Baumgardt, S.L.W. McMillan, J. Makino, P. Hut, T. Ebisuzaki, The Ecology of Star Clusters and Intermediate-Mass Black Holes in the Galactic Bulge. Astrophys. J. 641, 319-326 (2006). doi:10.1086/500361

K.A. Postnov, L.R. Yungelson, The Evolution of Compact Binary Star Systems. Living Reviews in Relativity 9, 6 (2006)

F. Pretorius, Evolution of Binary Black-Hole Spacetimes. Physical Review Letters 95(12), 121101 (2005). doi:10.1103/PhysRevLett.95.121101

R.R. Rafikov, Structure and evolution of circumbinary disks around supermassive black hole (SMBH) binaries. ArXiv e-prints (2012)

P. Ranalli, A. Comastri, G. Setti, The $2-10 \mathrm{keV}$ luminosity as a Star Formation Rate indicator. Astron. Astrophys. 399, 39-50 (2003). doi:10.1051/0004-6361:20021600

M.J. Rees, Accretion and the quasar phenomenon. Phys. Scr 17, 193-200 (1978). doi:10.1088/0031-8949/17/3/010

M.J. Rees, Tidal disruption of stars by black holes of 10 to the 6 th-10 to the 8 th solar masses in nearby galaxies. Nature 333, 523-528 (1988). doi:10.1038/333523a0

J.A. Regan, M.G. Haehnelt, Pathways to massive black holes and compact star clusters in pre-galactic dark matter haloes with virial temperatures \& 10,000K. Mon. Not. Roy. Acad. Soc. 396, 343-353 (2009). doi:10.1111/j.1365-2966.2009.14579.x

Y. Rephaeli, E.E. Salpeter, Flow past a massive object and the gravitational drag. Astrophys. J. 240, 20-24 (1980). doi:10.1086/158202

C. Rodriguez, G.B. Taylor, R.T. Zavala, A.B. Peck, L.K. Pollack, R.W. Romani, A Compact Supermassive Binary Black Hole System. Astrophys. J. 646, 49-60 (2006). doi:10.1086/504825

C. Roedig, O. Zanotti, D. Alic, General relativistic radiation hydrodynamics of accretion flows - II. Treating stiff source terms and exploring physical limitations. Mon. Not. Roy. Acad. Soc. 426, 1613-1631 (2012). doi:10.1111/j.1365-2966.2012.21821.x

N. Roos, J.S. Kaastra, C.A. Hummel, A massive binary black hole in $1928+738$ ? Astrophys. J. 409, 130-133 (1993). doi:10.1086/172647

S. Rosswog, E. Ramirez-Ruiz, W.R. Hix, Tidal Disruption and Ignition of White Dwarfs by 
Moderately Massive Black Holes. Astrophys. J. 695, 404-419 (2009). doi:10.1088/0004$637 \mathrm{X} / 695 / 1 / 404$

M. Ruffert, Three-dimensional hydrodynamic Bondi-Hoyle accretion. 1: Code validation and stationary accretors. Astrophys. J. 427, 342-350 (1994). doi:10.1086/174144

M. Ruffert, Non-axisymmetric wind-accretion simulations. II. Density gradients. Astron. Astrophys. 346, 861-877 (1999)

M. Saijo, T.W. Baumgarte, S.L. Shapiro, M. Shibata, Collapse of a Rotating Supermassive Star to a Supermassive Black Hole: Post-Newtonian Simulations. Astrophys. J. 569, 349-361 (2002). doi:10.1086/339268

A.P. Schoenmakers, A.G. de Bruyn, H.J.A. Röttgering, H. van der Laan, C.R. Kaiser, Radio galaxies with a 'double-double morphology' - I. Analysis of the radio properties and evidence for interrupted activity in active galactic nuclei. Mon. Not. Roy. Acad. Soc. 315, 371-380 (2000). doi:10.1046/j.1365-8711.2000.03430.x

N.I. Shakura, R.A. Sunyaev, Black holes in binary systems. Observational appearance. Astron. Astroph. 24, 337-355 (1973)

S.L. Shapiro, S.A. Teukolsky, Black Holes, White Dwarfs, and Neutron Stars: The Physics of Compact Objects, in 14.2. Collisionless Spherical Accretion, 1983a

S.L. Shapiro, S.A. Teukolsky, Black Holes, White Dwarfs, and Neutron Stars: The Physics of Compact Objects, in 12. Black Holes (eq. 12.4.30 and 12.7.25), 1983b

S.L. Shapiro, S.A. Teukolsky, Black Holes, White Dwarfs, and Neutron Stars: The Physics of Compact Objects 1983c

Y. Shen, A. Loeb, Identifying Supermassive Black Hole Binaries with Broad Emission Line Diagnosis. Astrophys. J. 725, 249-260 (2010). doi:10.1088/0004-637X/725/1/249

J.-M. Shi, J.H. Krolik, S.H. Lubow, J.F. Hawley, Three-dimensional Magnetohydrodynamic Simulations of Circumbinary Accretion Disks: Disk Structures and Angular Momentum Transport. Astrophys. J. 749, 118 (2012). doi:10.1088/0004-637X/749/2/118

E. Shima, T. Matsuda, U. Anzer, G. Boerner, H.M.J. Boffin, Numerical computation of two dimensional wind accretion of isothermal gas. Astron. Astrophys. 337, 311-320 (1998)

N. Soker, Stability analysis of the accretion line. Astrophys. J. 358, 545-550 (1990). doi:10.1086/169007

N. Soker, Nonlinear instability of the accretion line. Astrophys. J. 376, 750-756 (1991). doi:10.1086/170322

N. Stone, A. Loeb, Observing Lense-Thirring Precession in Tidal Disruption Flares. Physical Review Letters 108(6), 061302 (2012a). doi:10.1103/PhysRevLett.108.061302

N. Stone, A. Loeb, Tidal disruption flares of stars from moderately recoiled black holes. Mon. Not. Roy. Acad. Soc. 422, 1933-1947 (2012b). doi:10.1111/j.1365-2966.2012.20577.x

N. Stone, R. Sari, A. Loeb, Consequences of Strong Compression in Tidal Disruption Events. ArXiv e-prints (2012)

L.E. Strubbe, E. Quataert, Optical flares from the tidal disruption of stars by massive black holes. Mon. Not. Roy. Acad. Soc. 400, 2070-2084 (2009). doi:10.1111/j.13652966.2009.15599.x

H. Sudou, S. Iguchi, Y. Murata, Y. Taniguchi, Orbital Motion in the Radio Galaxy 3C 66B: Evidence for a Supermassive Black Hole Binary. Science 300, 1263-1265 (2003). doi:10.1126/science. 1082817

T. Tanaka, Z. Haiman, The Assembly of Supermassive Black Holes at High Redshifts. Astrophys. J. 696, 1798-1822 (2009). doi:10.1088/0004-637X/696/2/1798

A. Tchekhovskoy, B.D. Metzger, D. Giannios, L.Z. Kelley, Swift J1644+57 gone MAD: the case for dynamically-important magnetic flux threading the black hole in a jetted tidal disruption event. ArXiv e-prints (2013)

K.S. Thorne, A.N. Zytkow, Stars with degenerate neutron cores. I - Structure of equilibrium models. Astrophys. J. 212, 832-858 (1977). doi:10.1086/155109

M. Ugliano, H.-T. Janka, A. Marek, A. Arcones, Progenitor-explosion Connection and Remnant Birth Masses for Neutrino-driven Supernovae of Iron-core Progenitors. Astrophys. J. 757, 69 (2012). doi:10.1088/0004-637X/757/1/69

S. van Velzen, G.R. Farrar, The Rate of Stellar Tidal Disruption Flares from Sdss Data, in European Physical Journal Web of Conferences. European Physical Journal Web of Conferences, vol. 39, 2012, p. 8002. doi:10.1051/epjconf/20123908002

E.P. Velikhov J. Exp. Theor. Phys. 36, 1398 (1959)

J. Wang, D. Merritt, Revised Rates of Stellar Disruption in Galactic Nuclei. Astrophys. J. 600, 149-161 (2004). doi:10.1086/379767 
N. Webb, D. Cseh, E. Lenc, O. Godet, D. Barret, S. Corbel, S. Farrell, R. Fender, N. Gehrels, I. Heywood, Radio Detections During Two State Transitions of the Intermediate-Mass Black Hole HLX-1. Science 337, 554 (2012). doi:10.1126/science.1222779

J.S.B. Wyithe, A. Loeb, Low-Frequency Gravitational Waves from Massive Black Hole Binaries: Predictions for LISA and Pulsar Timing Arrays. Astrophys. J. 590, 691-706 (2003). doi: $10.1086 / 375187$

J.S.B. Wyithe, A. Loeb, Detection of Gravitational Waves from the Coalescence of Population III Remnants with Advanced LIGO. Astrophys. J. 612, 597-601 (2004). doi: $10.1086 / 422183$

J.S.B. Wyithe, A. Loeb, Photon trapping enables super-Eddington growth of black hole seeds in galaxies at high redshift. Mon. Not. Roy. Acad. Soc. 425, 2892-2902 (2012). doi:10.1111/j.1365-2966.2012.21127.x

O. Zanotti, C. Roedig, L. Rezzolla, L. Del Zanna, General relativistic radiation hydrodynamics of accretion flows - I. Bondi-Hoyle accretion. Mon. Not. Roy. Acad. Soc. 417, 2899-2915 (2011). doi:10.1111/j.1365-2966.2011.19451.x 